\title{
Transcriptional Regulation of the GluR2 Gene: Neural-Specific Expression, Multiple Promoters, and Regulatory Elements
}

\author{
Scott J. Myers,, ${ }^{1,2}$ Jeanne Peters, ${ }^{1}$ Yunfei Huang, ${ }^{1}$ Mary B. Comer, ${ }^{2}$ Fabrice Barthel, ${ }^{3}$ and \\ Raymond Dingledine ${ }^{1}$ \\ ${ }^{1}$ Department of Pharmacology, Emory University, Atlanta, Georgia 30322, 2Department of Pharmacology, University of \\ North Carolina at Chapel Hill, Chapel Hill, North Carolina 27599, ${ }^{3}$ U 259 INSERM, Universite de Bordeaux II, 33077 \\ Bordeaux Cedex, France
}

To understand how neurons control the expression of the AMPA receptor subunit GluR2, we cloned the 5' proximal region of the rat gene and investigated GluR2 promoter activity by transient transfection. RNase protection and primer extension of rat brain mRNA revealed multiple transcription initiation sites from -340 to -481 bases upstream of the GluR2 AUG codon. The relative use of $5^{\prime}$ start sites was different in cortex and cerebellum, indicating complexity of GluR2 transcript expression among different sets of neurons. When GluR2 promoter activity was investigated by plasmid transfection into cultured cortical neurons, cortical glia, and C6 glioma cells, the promoter construct with the strongest activity, per transfected cell, was 29.4-fold ( \pm 3.7) more active in neurons than in non-neural cells. Immunostaining of cortical cultures showed that $>97 \%$ of the luciferase-positive cells also expressed the neuronal marker MAP-2. Evaluation of internal deletion and substitution mutations identified a functional repressor element I RE1-like silencer and functional Sp1 and nuclear respiratory factor-1
(NRF-1) elements within a GC-rich proximal GluR2 promoter region. The GluR2 silencer reduced promoter activity in glia and non-neuronal cell lines by two- to threefold, was without effect in cortical neurons, and could bind the RE1-silencing transcription factor (REST) because cotransfection of REST into neurons reduced GluR2 promoter activity in a silencer-dependent manner. Substitution of the GluR2 silencer by the homologous Nall RE1 silencer further reduced GluR2 promoter activity in nonneuronal cells by $30-47 \%$. Maximal positive GluR2 promoter activity required both $\mathrm{Sp} 1$ and NRF-1 cis elements and an interelement nucleotide bridge sequence. These results indicate that GluR2 transcription initiates from multiple sites, is highly neuronal selective, and is regulated by three regulatory elements in the $5^{\prime}$ proximal promoter region.

Key words: AMPA; glutamate receptor; transcription; REST; NRF-1; primary culture; transfection; luciferase; neurons; promoter; Sp1; silencer; neuronal expression; repressor
The AMPA subtypes of glutamate receptors are assembled from combinations of GluR1, 2, 3 and 4 (GluRA, B, C, D) subunits and mediate a large fraction of the postsynaptic response at most fast excitatory synapses in the brain. Studies with recombinant receptors indicate that different subunit combinations produce functionally unique AMPA receptors (Boulter et al., 1990; Sommer et al., 1990; Hollmann et al., 1991; Lomeli et al., 1994). In particular, three AMPA receptor properties are strongly influenced by the level of edited GluR2 expression because of the positively charged arginine present in the $\mathrm{Q} / \mathrm{R}$ site of the $\mathrm{M} 2$ channel-lining domain. The presence of edited GluR2 reduces $\mathrm{Ca}^{2+}$ permeability (Hollmann et al., 1991; Geiger et al., 1995), voltage-dependent channel block by cytosolic polyamines (Bowie and Mayer, 1995; Donevan and Rogawski, 1995; Kamboj et al., 1995; Koh et al., 1995), and single channel conductance (Swanson et al., 1997). Thus, EPSPs mediated by GluR2-deficient receptors may be

\footnotetext{
Received Feb. 13, 1998; revised June 17, 1998; accepted June 19, 1998.

This work was supported by a National Institutes of Health (NIH) grant (R.D.), an NIH individual predoctoral fellowship (S.J.M.), and an unrestricted grant from Bristol-Myers Squibb (R.D.). We are indebted to Dr. Gail Mandel for the generous gift of the Type II Na channel promoter and RESTexpress vectors and Dr. Richard Scarpulla for the NRF-1 expression vector. We thank Sunan Zhang for excellent technical assistance with the neuronal cultures and Warren Hauk and Nell Birkhahn for assistance in DNA sequencing.

Correspondence should be addressed to Dr. Scott J. Myers, Department of Pharmacology, Emory University School of Medicine, Atlanta, GA 30322.

Copyright (C) 1998 Society for Neuroscience $0270-6474 / 98 / 186723-17 \$ 05.00 / 0$
}

larger than those mediated by GluR2-rich receptors, which should have significant physiological consequences.

The relative expression of AMPA receptor subunit mRNA and protein in certain populations of neurons is not static but may be remodeled during development (Pellegrini-Giampietro et al., 1992a), after seizures or ischemic insult (Pellegrini-Giampietro et al., 1992b, 1994; Pollard et al., 1993; Freidman et al., 1994; Kamphuis et al., 1994; Prince et al., 1995) and after administration of antipsychotics (Fitzgerald et al., 1995), drugs of abuse (Ortiz et al., 1995; Fitzgerald et al., 1996), or corticosteroids (Nair et al., 1998). After ischemia, a drop in relative GluR2 expression in hippocampal CA1 pyramidal cells is associated with changes in AMPA receptor properties consistent with an increase in GluR2deficient receptors (Gorter et al., 1997), although whether GluR2 selectively declines remains controversial (see Frank et al., 1995). Recent work from our laboratory indicates that the number of GluR2 subunits in a receptor is variable and that the relative abundance of GluR2 affects $\mathrm{Ca}^{2+}$ permeability and rectification differentially (Washburn et al., 1997). Given that distinct populations of neurons are known to be GluR2-deficient or GluR2rich (Bochet et al., 1994; Jonas et al., 1994; Geiger et al., 1995; Washburn et al., 1997), the consequences of modulating GluR2 expression in these cells will depend on pre-existent GluR2 levels.

These studies indicate that mechanisms controlling the level of GluR2 expression may be important regulatory determinants of AMPA receptor phenotype. GluR2 expression in brain is pri- 
marily restricted to neurons (Monyer et al., 1991; Petralia and Wenthold, 1992; Sato et al., 1993), although AMPA receptor subunits are expressed by certain populations of glia (Keinänen et al., 1990; Burnashev et al., 1992) and O-2A progenitor cells (Patneau et al., 1994). Functional promoter studies of NMDA and kainate receptor genes have been reported (Bai and Kusiak, 1995; Sasner and Buonanno, 1996; Huang and Gallo, 1997; Suchanek et al., 1997), and the organization of the mouse GluR2 gene has been described (Köhler et al., 1994), but no regulatory elements of AMPA receptor subunits have yet been identified. We show here that the GluR2 proximal promoter region contains a negative regulatory element and a positive regulatory region and directs the neural-specific expression of a luciferase reporter gene in transiently transfected primary cortical cultures.

Parts of this paper have been published previously in abstract form (Peters et al., 1995; Myers et al., 1996).

\section{MATERIALS AND METHODS}

Materials. Rat C6 glioma (CCL-107) cells were obtained from American Type Culture Collection (Rockville, MD). HeLa cells were a gift from Dr. T. J. Murphy (Emory University). Timed-pregnant rats were purchased from Harlan Sprague Dawley (Indianapolis, IN). DMEM and MEM tissue culture media, L-glutamine, trypsin, and EDTA were obtained from Life Technologies (Gaithersburg, MD). Poly-D-lysine (molecular weight of $>60,000)$, fetal bovine serum, and tissue culture supplements human transferrin, putrescine, tri-iodothyronine, progesterone, estradiol, sodium selenite, insulin, L-glucose, and sodium bicarbonate were obtained from Sigma (St. Louis, MO). Mouse monoclonal anti-MAP-2 (clone AP20) was purchased from Boehringer Mannheim (Indianapolis, IN), mouse monoclonal anti-GFAP (clone G-A-5) was from Sigma, polyclonal rabbit anti-luciferase was from Promega (Madison, WI), and Texas Red-conjugated $\mathrm{F}\left(\mathrm{ab}^{\prime}\right)_{2}$ donkey anti-rabbit IgG and FITC-conjugated $\mathrm{F}\left(\mathrm{ab}^{\prime}\right)_{2}$ donkey anti-mouse IgG were from Jackson ImmunoResearch (West Grove, PA). Anti-Sp1 and anti-Krox-24 antibodies and $\mathrm{Sp} 1$ and Krox-24 double-stranded oligonucleotides were purchased from Santa Cruz Biotechnology (Tebu, France).

$P f u$ polymerase and the $\lambda$ DASH II genomic library were from Stratagene (La Jolla, CA). Lipofectamine, Superscript II reverse transcriptase, and RNAsin were obtained from Life Technologies. MMLV reverse transcriptase was from Stratagene. Restriction enzymes were purchased from New England Biolabs (Beverly, MA) or Life Technologies. T4 DNA ligase, T4 DNA and Klenow polymerases, RNaseONE, luciferase vectors, and assay reagents were purchased from Promega. pcDNA3 and TA cloning vectors were from Invitrogen (San Diego, CA), and Sequenase 2.0 was purchased from United States Biochemicals (Cleveland, OH). Geneclean kits were obtained from BIO 101 (La Jolla, CA). All oligonucleotides were synthesized in house on a MilliGen/ Biosearch Cyclone Plus DNA Synthesizer using Expedite Chemistry (PerSeptive Biosystems). Radioisotopes were purchased from Amersham (Arlington Heights, IL). Nylon-backed nitrocellulose membranes were purchased from Bio-Rad (Hercules, CA). Other general salts and supplies were purchased from Sigma or Fisher Scientific (Houston, TX).

Isolation and characterization of genomic clones. Approximately $0.7 \times$ $10^{6}$ plaques from a rat genomic library in $\lambda$ DASH II were hybridized on nylon membranes with a 3505 bp EcoRI-XhoI restriction fragment containing the full coding sequence of the rat GluR2 cDNA, labeled with $\left[\alpha-{ }^{32} \mathrm{P}\right] \mathrm{dCTP}$ by random priming. Three positive plaques were purified and subjected to a second round of hybridization with an end-labeled oligonucleotide (R2-1; GAGGACAGAAATATGCATAATCTTTTGCAT) complementary to the first 10 codons of the GluR2 coding sequence (Boulter et al., 1990). A single recombinant phage obtained from this screen had an $18 \mathrm{~kb}$ insert that included a long, interspersed repeat segment (LINE-1) at its immediate $5^{\prime}$ end, $\sim 9 \mathrm{~kb}$ upstream of the $5^{\prime}$ end of the GluR2 gene. A $1.64 \mathrm{~kb} X b a \mathrm{I}$-HindIII fragment, which contained the GluR2 proximal promoter region, exon 1, and 288 bp of the first intron, has been deposited in the GenBank database (accession number AF025917).

$5^{\prime}$ RACE, RNase protection, and primer extension and Southern blot. For 5' RACE, poly $\left(\mathrm{A}^{+}\right)$mRNA isolated from adult male Sprague Dawley rat brains by the method of Chirgwin et al. (1979) was reverse transcribed by MMLV reverse transcriptase from the R2-1 primer. After addition of an oligo-dC tail with terminal deoxynucleotidyltransferase, PCR reactions were performed using the anchor primer provided by the Life Technologies 5' RACE system, plus the R2-race1 primer (AGTCCCGAGGACTGGCTGAA) complementary to nucleotides $81-100$ of the GluR2 cDNA sequence (Boulter et al., 1990). PCR products were subcloned into the TA cloning vector and selected by colony hybridization to the R2-20 oligonucleotide complementary to bp 64-83 of the GluR2 cDNA (GAATGCAGTTTTTAGCTGAG). Ten clones were selected by size on agarose gels and sequenced through the anchor-priming site to locate $5^{\prime}$ ends of the mRNA.

For primer extension, an oligonucleotide (PER2-3; TCCCAGTTGTAGCTGGTGGCTGTTGATGCC) complementary to bp -163 to -192 from the coding AUG (see Fig. $1 A$ ) was end-labeled with $\left[\gamma^{-32} \mathrm{P}\right]$ ATP $\left(\sim 4.8 \times 10^{5} \mathrm{cpm} / \mathrm{pmol}\right)$ and annealed to $30 \mu \mathrm{g}$ of adult rat cortical and cerebellar total RNA in $20 \mathrm{~mm}$ HEPES, pH 7.0, $350 \mathrm{~mm}$ $\mathrm{NaCl}$, and $50 \%$ formamide at $50^{\circ} \mathrm{C}$ for $24 \mathrm{hr}$. Annealed samples were precipitated and resuspended in $50 \mu$ l containing $50 \mathrm{~mm}$ Tris- $\mathrm{HCl}, \mathrm{pH}$ $8.3,75 \mathrm{~mm} \mathrm{KCl}, 3 \mathrm{~mm} \mathrm{MgCl} 2,2.5 \mathrm{~mm}$ dNTPs, $10 \mathrm{~mm}$ DTT, $50 \mu \mathrm{g} / \mathrm{ml}$ actinomycin D, 20 units RNAsin, and 200 units Superscript II reverse transcriptase and were incubated at $42^{\circ} \mathrm{C}$ for $90 \mathrm{~min}$; then $1 \mu \mathrm{l}$ of RNAsONE was added, and the incubation continued for an additional 35 min. Samples were recovered and loaded onto a sequencing gel. Yeast total RNA $(48 \mu \mathrm{g})$ and an in vitro synthesized full-length GluR2 sense RNA (10 ng) were used as templates in negative and positive control reactions, respectively. The in vitro GluR2 RNA includes 481 bases of GluR2 5'-untranslated region (UTR) sequence plus an additional $40 \mathrm{bp}$ of polylinker sequence. To confirm the specificity of the primer extension reactions, we also prepared Southern blots of primer extension products. For these experiments the reaction conditions were identical to those described above except that the oligonucleotide primer was unlabeled and, after gel resolution, reaction products were electrotransferred to a nylon membrane. Southern blots were then hybridized with a ${ }^{32} \mathrm{P}$ radiolabeled DNA probe $\left(>10^{8} \mathrm{cpm} / \mu \mathrm{g}\right)$ made from $\mathrm{bp}-192$ through -481 relative to the GluR2 AUG (see Fig. $2 A$, probe B). Hybridized blots were washed under high-stringent conditions and exposed to a Molecular Dynamics PhosphorImager plate (Sunnyvale, CA) for visualization.

RNase protection experiments were conducted using an Ambion RPA kit (Austin, TX) following the manufacturer's instructions. For identification of mRNA 5' ends, an antisense riboprobe complementary to nucleotides -192 through -481 relative to the GluR2 AUG (see Fig. $2 A$, probe $\mathrm{C})$ was radiolabeled and annealed $\left(5 \times 10^{5} \mathrm{cpm}\right.$ per reaction) to $30 \mu \mathrm{g}$ of adult rat cortical and cerebellar total RNA in $20 \mu \mathrm{l}$ of hybridization solution (Ambion kit) at $45^{\circ} \mathrm{C}$ overnight. Annealed samples were digested with RNase A and RNaseT1, resolved on an RNA-sequencing gel, and exposed to Kodak X-OMAT film for $12 \mathrm{hr}$. To quantify the relative use of transcription initiation sites in cerebellum and cortex, an antisense RNA probe complementary to bases -136 through -361 relative to the GluR2 AUG (see Fig. $2 A$, probe D) was annealed to total RNA as described above. This probe identifies the major short transcription initiation site at -340 and also summates all transcripts with $5^{\prime}$ ends extending beyond -360 into a single band on the gel. Total RNA used in these experiments was prepared independently from four adult male Sprague Dawley rats.

Methylation sites in the promoter region. Adult rat genomic DNA was isolated from lung, kidney, cortex, and cerebellum $(40-50 \mu \mathrm{g})$ and digested with $A c c$ I to release a $1.3 \mathrm{~kb}$ promoter fragment $(-731$ to +603 bp relative to the +1 transcription initiation site) and then was digested again with one of the methylation-sensitive restriction enzymes BssHII, SacII, $A v a \mathrm{I}$, or Eco47III. Southern blots of the genomic fragments were prepared and hybridized with an $A c c$ I-restricted $1.3 \mathrm{~kb}$ genomic fragment radiolabeled with $\left[\alpha_{-}{ }^{32} \mathrm{P}\right] \mathrm{dCTP}$ by random priming $\left(>10^{8} \mathrm{cpm} / \mu \mathrm{g}\right)$. All hybridizations and washes were conducted under high-stringent conditions.

Cell culture. Primary rat cortical neuronal cultures were prepared from embryonic day 18 fetal rat pups resected from pregnant Sprague Dawley rats. Briefly, cerebral cortices were minced in DMEM supplemented with $1.1 \mathrm{gm} / 1$ glucose, $2.1 \mathrm{gm} / 1 \mathrm{NaHCO}_{3}$, and $1.84 \mathrm{~mm}$ L-glutamine, $\mathrm{pH}$ 7.25 (FDMEM); $1 \mathrm{ml}$ of fetal bovine serum (FBS) was added; and tissue was dissociated by trituration. Dissociated cells were rinsed in $8 \mathrm{ml}$ of FDMEM media, pelleted at low speed for $5 \mathrm{~min}$, and then resuspended in FDMEM supplemented with $100 \mu \mathrm{g} / \mathrm{ml}$ human apotransferrin and (in $\mu \mathrm{M}$ ): insulin 0.5 , putrescine 60 , tri-iodothyronine $1 \times 10^{-3}$, $\beta$-estradiol $1 \times 10^{-6}$, progesterone 0.01 , and sodium selenite 0.03 (termed TFDMEM). Cortical cells were plated at a density of $0.7-1.0 \times$ 
$10^{6}$ cells/well into Falcon 12-well culture dishes precoated overnight with $180 \mu \mathrm{g} / \mathrm{ml}$ poly-D-lysine and coated with $20 \%$ FBS in FDMEM for 2-3 $\mathrm{hr}$ before plating. Cultures were grown for 3-6 d in vitro, without media change, in an humidified, $5 \% \mathrm{CO}_{2}, 37^{\circ} \mathrm{C}$ incubator. It should be noted that these cultures are a mixture of neurons and glia with $\sim 60 \%$ of cells MAP-2 positive on days 4-6 in culture.

Primary glial cultures were generated from the primary cortical neuronal cultures by repeated washing of cells with FDMEM plus 10\% FBS over a period of 1-4 weeks after plating or by passage of cultures to facilitate the elimination of neurons. Cortical glia and rat C6 glioma cells were grown in FDMEM plus $10 \%$ FBS until use in transfections. HeLa cells were grown in DMEM supplemented with $10 \%$ FBS and $2 \mathrm{~mm}$ glutamine. The non-neuronal cultures were grown in the same incubator as the neurons.

Constructs and mutagenesis. To generate 5'- and $3^{\prime}$-GluR2 promoter deletion constructs, we amplified specific-length fragments of the GluR2 promoter by PCR with $P f u$ polymerase using oligonucleotides complementary to the GluR2 $1.64 \mathrm{~kb} X b a \mathrm{I}-H i n d I I I$ GluR2 fragment. 5'- and 3 '-oligonucleotides contained NheI and BglII restriction sites, respectively. Amplified DNA fragments were restricted and cloned into the NheI and BglII sites of the pGL2 basic vector upstream of the reporter gene firefly luciferase. Recombinants were analyzed by restriction and sequence analysis to confirm the location of the GluR2 promoter boundaries. Throughout this report, construct boundaries [i.e., R2 $(-822 /$ $+320) l u c]$ are defined relative to the $5^{\prime}$-most major initiation site identified, -429 bases upstream from the recognized GluR2 AUG.

To generate internal GluR2 silencer mutations in the context of the $\mathrm{R} 2(-302 /+320) l u c$ construct, we used a two-round PCR mutagenesis protocol as described (Cormack, 1997). After the second-round PCR reaction, the mutagenized DNA fragment was restricted with NheI and $B g l \mathrm{II}$ and cloned back into the pGL2 basic vector. To delete the GluR2 silencer, we replaced 25 bp spanning the silencer element with an NsiI restriction site. This construct is designated $\mathrm{R} 2(-302 /+320)$ luc $-\Delta \mathrm{B}$. Substitution mutations were introduced by replacing different 6 bp segments of the silencer with either an EcoRI or an EcoRV restriction site and were designated $\mathrm{R} 2(\delta \mathrm{RI}) l u c$ and $\mathrm{R} 2(\delta \mathrm{RV}) l u c$, respectively. Constructs that replaced the GluR2 silencer with the Type II Na channel repressor element 1 (NaII-RE1) silencer in the context of the GluR2 promoter $[\mathrm{R} 2(\delta \mathrm{NaII}) l u c]$, or likewise replaced the NaII-RE1 element with the GluR2 silencer in the context of the NaII promoter $[\mathrm{NaII}(\delta \mathrm{R} 2) l u c]$, were also made using the two-round, oligonucleotide-directed PCR mutagenesis protocol. The NaII promoter construct without an RE1 element (pMB4) (Kraner et al., 1992) was excised with EcoRI, blunted with Klenow, cloned into the SmaI-digested pGL2 basic vector, and renamed $\mathrm{NaII}(\Delta) l u c$. The NaII promoter construct retaining the RE1 silencer on a $118 \mathrm{bp}$ fragment $(-1051$ to -933$)$ fused $5^{\prime}$ of the NaII minimal promoter region $(-134$ to +177$)$ as described (pSDK7) (Kraner et al., 1992) and was cloned upstream of firefly luciferase by shuttling it through the pBluescript HindIII-PstI polylinker sites and into the pGL2 basic vector KpnI and NheI sites. This construct was renamed NaIIluc. All constructs described were confirmed by restriction and sequence analysis and preserved the correct flanking sequences and silencer orientations of their natural promoter contexts; e.g., R2( $\delta \mathrm{NaII}) l u c$ contains type II sodium channel RE1 silencer with GluR2 flanking sequence and the GluR2 antisense orientation. The pairs of oligonucleotides used to introduce these mutations are the following: to delete the silencer in GluR2, R2 $(-302 /+320) l u c-\Delta \mathrm{B}$, sense TCCGACTATGCATCGGATGCGCAACAC and antisense GCATCCGATGCATAGTCGGAGCTCTGG; the missense mutation R2( $\delta \mathrm{RI}) l u c$, sense AAAGCGCTGTGAATTCTGCTAAAATCGGATGCG and antisense CCGATTTTAGCAGAATTCACAGCGCTTTAGTCGG; the missense mutation R2( $\delta \mathrm{RV}) l u c$, sense CTAAAGGATATCCCTCGGTGCTAAAATCGG and antisense CCGAGGGATATCCTTTAGTCGGAGCTC; to place the NaII RE1 silencer in the GluR2 promoter, R2 $(\delta \mathrm{NaII}) l u c$, sense TGCTGTCCGTGGTTCTGAAATCGGAT and antisense AGAACCACGGACAGCACTTTAGTCG; to place the GluR2 silencer in the NaII promoter, NaII $(\delta \mathrm{R} 2) l u c$, sense GCACCGAGGACAGCGCTAGAGTCTCTGAAT and antisense GCTGTCCTCGGTGCTAAAACCCAATTACAG.

A two-round PCR mutagenesis strategy was also used to introduce systematically internal deletions of $25-30$ bp in the R2 $(-302 /+320) l u c$ construct to generate a "deletion scan" of the proximal region of the GluR2 promoter. For each new mutagenized construct, a unique $6 \mathrm{bp}$ restriction $(E c o \mathrm{RV}, N s i \mathrm{I}$, or $S t u \mathrm{I})$ site was introduced to allow easy identification of recombinants. All mutant constructs were sequenced for verification. The designations for the internal deletion mutants are $\mathrm{R} 2(-302 /+320) l u c-\Delta \mathrm{A}$ through $\Delta \mathrm{N}$, and the following GluR2 promoter regions are deleted: $\Delta \mathrm{A},-227$ to $-197 ; \Delta \mathrm{B},-196$ to $-172 ; \Delta \mathrm{C},-171$ to $-147 ; \Delta \mathrm{D},-146$ to $-118 ; \Delta \mathrm{E},-117$ to $-90 ; \Delta \mathrm{F},-89$ to $-65 ; \Delta \mathrm{G},-64$ to $-40 ; \Delta \mathrm{H},-39$ to $-15 ; \Delta \mathrm{I},-14$ to $+14 ; \Delta \mathrm{J},+15$ to $+38 ; \Delta \mathrm{K},-3$ to $+74 ; \Delta \mathrm{L},+71$ to $+146 ; \Delta \mathrm{M},+107$ to $+206 ;$ and $\Delta \mathrm{N},+182$ to +206 . Oligonucleotide sequences used to introduce internal mutations for the $\mathrm{R} 2(-302 /+320)$ luc deletion scan are available on request.

Transfections. Primary neurons were transfected on days 3-6 after plating. Primary glia were transfected either as confluent, unpassaged cells or at $90 \%$ confluency $2-3 \mathrm{~d}$ after passage, and C6 glioma cells were transfected similarly at $80-100 \%$ confluency. All transfections were conducted in Falcon 12-well dishes with Lipofectamine according to the manufacturer's instructions. Per well, $1 \mu \mathrm{g}$ of DNA, $50 \mu \mathrm{l}$ of $150 \mathrm{~mm}$ $\mathrm{NaCl}$, and $5 \mu \mathrm{l}$ of Lipofectamine were combined in $0.5 \mathrm{ml}$ of TFDMEM media and incubated with cells at $37^{\circ} \mathrm{C}$ for $5 \mathrm{hr}$. After transfection, the DNA and Lipofectamine mixture was replaced with fresh TFDMEM $(0.5 \mathrm{ml})$ for primary neuronal cultures or with normal culture media for primary glia, C6 glioma, and HeLa cells. Twenty-four hours later, cells were rinsed with PBS and harvested by lysis. Cell lysates were cleared by centrifugation and assayed for luciferase activity in a Turner TD-20e luminometer. A preliminary time course study with the GluR2, cytomegalovirus (CMV), and SV40 promoters in transfected cortical cultures indicated that luciferase expression was maximal $24 \mathrm{hr}$ after transfection and decreased slowly by 48 and $72 \mathrm{hr}$.

For cotransfections, a similar protocol was used except the amount of Lipofectamine was increased to $7.5 \mu \mathrm{l}$ per well to accommodate the additional DNA. The amount of the GluR2 promoter-firefly reporter construct was varied from 0.1 to $1.0 \mu \mathrm{g} /$ well, and the amount of the RE1-repressor factor plasmid (RESTexpress) (Chong et al., 1995) or the control plasmid pcDNA3 was held fixed at 0.25 or $0.75 \mu \mathrm{g} /$ well, and pBS vector was added to adjust the total DNA for all transfections to a final concentration of $1.75 \mu \mathrm{g} /$ well. Under these cotransfection conditions, the amount of luciferase activity recovered in the cell lysates varied linearly with the amount of pGL2 reporter plasmid DNA transfected (data not shown). For nuclear respiratory factor-1 (NRF-1) cotransfections, $1 \mu \mathrm{g}$ of pNRF-1 plasmid (Virbasius et al., 1993) was mixed with $0.5 \mu \mathrm{g}$ of the GluR2 promoter-reporter construct

All plasmid DNA preparations were prepared from $100 \mathrm{ml}$ of $2 \mathrm{xYT}$ cultures using Qiagen Maxi-DNA Prep columns. At least three independent DNA preparations for each construct were transfected, in parallel, in each experiment. Each individual DNA plasmid was transfected into triplicate wells. For transfections into multiple cell types, a master mix of the plasmid DNA, NaCl, Lipofectamine, and TFDMEM media was prepared from which equal aliquots were delivered to the cells. In all experiments, an SV40-luciferase or a CMV-luciferase control plasmid was transfected into parallel wells to normalize GluR2 promoter activity across multiple experiments and primary culture preparations. Where indicated, luciferase activity was converted to moles of luciferase $(\sim 2.2$ Turner light units $/ 10^{-18}$ mole) using a standard curve generated with recombinant firefly luciferase under our assay conditions.

Immunofluorescence. Primary neurons prepared as described above were plated onto glass coverslips precoated with both poly-D-lysine (overnight) and then fibronectin $(20 \mu \mathrm{g} / \mathrm{ml} ; 1-2 \mathrm{hr})$. Cultures were transfected with the R2 $(-302 /+320)$ luc or the R2 $(-134 /+147)$ luc construct and fixed $24 \mathrm{hr}$ later with $4 \%$ paraformaldehyde in PBS for $15 \mathrm{~min}$. Cells were rinsed three times with PBS, permeabilized with $0.1 \% \mathrm{w} / \mathrm{v}$ Triton X-100 in PBS for 5 min, washed, and then incubated in staining buffer (2\% horse serum in PBS) for 15 min. Cells were incubated for $2 \mathrm{hr}$ in staining buffer with rabbit anti-luciferase (1:200) and either mouse anti-GFAP (1:800) or mouse anti-MAP-2 (1:600) and then rinsed four times for 5 min each with excess staining buffer before incubation with the secondary antibodies, Texas Red-conjugated donkey anti-mouse IgG (1:250) and FITC-conjugated donkey anti-rabbit $\operatorname{IgG}(1: 250)$, in staining buffer for $30 \mathrm{~min}$. Cells were rinsed four times for 5 min each as described above and mounted onto glass slides. All washes and staining incubations were conducted at room temperature. Fluorescence was visualized with a Zeiss Axioplan microscope using a $40 \times$ PlanNEOFLUAR oil objective and appropriate fluorescent filters. Cells positively identified for firefly luciferase expression were counted and scored for positivity to either MAP-2 or GFAP expression.

Nuclear extract and gel mobility shift assays. Nuclear extracts were prepared from cultured cells according to the method of Dignam et al. (1983). For gel mobility shift assays, complementary oligonucleotides were purified by denaturing PAGE and then annealed and ${ }^{32} \mathrm{P}$-labeled by 
Klenow fill-in of the $5^{\prime}$ overhangs for $25 \mathrm{~min}$ at room temperature. Labeled probes were extracted, purified through a spin column, precipitated, and stored in deionized water at $4^{\circ} \mathrm{C}$ until use.

In vitro binding reactions were conducted in $20 \mu \mathrm{l}$ volumes containing (final concentrations) 12 mM HEPES, pH 7.9, 4 mM Tris-HCl, pH 7.9, 60 mM KCl, 1 mM EDTA, 12\% v/v glycerol, 1 mM DTT, 1 mM PMSF, 0.225 $\mu \mathrm{g} / \mu \mathrm{l} \mathrm{BSA}, 0.1 \mu \mathrm{g} / \mathrm{ml}$ poly $\mathrm{dI}: \mathrm{dC}$, and 5-10 $\mu \mathrm{g}$ of nuclear extract protein. Individual components of the binding reaction were combined and incubated on ice for $15-30 \mathrm{~min}$ before the addition of probe $(\sim 0.3 \mathrm{nM}$, final concentration; $10-30 \times 10^{3} \mathrm{cpm}$ ) and then were incubated at $22^{\circ} \mathrm{C}$ for 15 min. Where indicated, a 30-200-fold excess of cold competitor oligonucleotides was added on ice 15-30 min before addition of probe. After incubation, samples were separated on a $4 \%$ polyacrylamide, Trisglycine, glycerol gel. Dried gels were exposed to Kodak X-OMAT film or to a Molecular Dynamics PhosphorImager plate for visualization.

Double-stranded oligonucleotide probes used in the binding reactions are the following (top strand): probe G, GGCGCTGTGCGGGGGAGGGGTAGGTGCGCGA; probe H, CTAGAGCTCCCTGCCTGCCTTGAGTCGGATC; Sp1 consensus, ATTCGATCGGGGCGGGGCGAGC; $\delta$ 1 mutant, GGCGCTGTGCTCTAGAGGGGTAGGTGCGCGA; and Krox-24 consensus, GGATCCAGCGGGGGCGAGCGGGGGCGA.

Statistical analysis. For comparison of the promoter activities between two or more constructs within a given cell type, at least three plasmid preparations for each construct were transfected in the same experiment. The order in which individual plasmid DNA preparations were added to the wells for transfection was randomized to reduce bias attributable to handling of the cells during the procedure. Raw luciferase activity was normalized to the activity of either the SV40 promoter or a defined control construct measured in parallel wells. Data from multiple experiments (culture preparations) were combined and analyzed by ANOVA followed by post hoc Dunnett's tests for significance between means or, when appropriate, Student's $t$ tests. Comparisons of the promoter activities between constructs were made within, and not across, cell types.

\section{RESULTS}

\section{Multiple tissue-specific transcription initiation sites and DNA methylation}

Exon 1 and the proximal $5^{\prime}$-flanking sequence of the rat GluR2 gene are shown in Figure $1 A$, along with a schematic identifying the salient features. To identify transcription initiation sites, we first conducted 5' RACE analysis of rat brain mRNA. Multiple potential initiation sites were identified from -286 to -481 bases upstream from the GluR2 translation AUG codon. Most RACE $5^{\prime}$ ends mapped to one of two clusters, from -286 to -295 or from -419 to $-427 \mathrm{bp}$, with one end residing at $-481 \mathrm{bp}$ (Fig. $1 A$, open triangles). The sequence of each RACE PCR product matched that of the genomic DNA up through the anchorpriming site, reducing the likelihood that an intron resides in the GluR2 5'-UTR, in accordance with previous observations for the mouse GluR2 gene (Köhler et al., 1994).

To further identify potential sites of transcription initiation in the GluR2 gene, we used both primer extension and RNase protection analysis of adult rat brain RNA (Fig. 2). Primer extension analysis on cortical RNA using an oligonucleotide, PER2-3 complementary to the GluR2 5'-UTR, revealed multiple reaction products extending from -312 to -452 nucleotides upstream from the GluR2 AUG (Fig. 2B). None of these cortical RNA primer extension products appeared in parallel reactions conducted with an in vitro synthesized genomic GluR2 RNA control $(\mathrm{Ctl})$ that includes putative 5 '-UTR sequence from -1 through -481 bases; this positive control also includes an additional $40 \mathrm{bp}$ of non-GluR2 polylinker sequence (Fig. 2B, left lane). This result indicates that the multiple extension products observed in cortical RNA reactions are not the result of RNA secondary structure preventing the processivity of reverse transcriptase. To determine which PER2-3 primed extension products from cortical RNA were derived from GluR2, a parallel primer extension reaction was run using unlabeled PER2-3 oligonucleotide as primer. Products from this reaction were then Southern blotted onto a nylon membrane and hybridized with probe B, DNA complementary to bases -192 through $-4815^{\prime}$ of the GluR2 AUG (Fig. 2A). The results of this Southern blot, depicted in the right lane of Figure $2 B$, confirm the identity of nine GluR2 primer extension products. A single reaction product at -312 was not confirmed by the Southern blot and is presumably an artifact caused by extension from a non-GluR2 transcript.

We also conducted RNase protection assays on rat cortical and cerebellar RNA to confirm the primer extension findings. Using an antisense RNA probe ( -192 through -481 bp of the GluR2 leader, probe $\mathrm{C}$ in Fig. $2 A$ ), we again detected multiple bands predicting different GluR2 5' ends in the cortex and cerebellum, and these bands were in agreement with results obtained by primer extension (Fig. 2C). Major RNase-protected 5' ends mapped to $-340,-368,-399$, and -429 bp $5^{\prime}$ of the GluR2 AUG codon, with additional minor ends mapping to -452 and -481 bp. Because the riboprobe in this experiment included GluR2 sequence only to position $-481 \mathrm{bp}$, the -481 band observed at the top of the gel (Fig. $2 C, C b$ and $C t x$ lanes) is a summation of all natural GluR2 transcripts with $5^{\prime}$ leaders at least this long. Despite this, however, the intensity of the -481 band was much weaker than that observed for shorter, more dominant mRNA species, indicating that long transcripts were few in number. From these analyses, it seems that GluR2 initiates transcription from multiple dominant sites between -340 and -429 bases upstream of the GluR2 AUG, with a minority of transcripts initiating at -452 and -481 bases. Therefore, depending on the site of transcription initiation, GluR2 mRNAs will contain a variable-length and unusually long 5'-UTR leader sequence (Kozak, 1987), a GC-rich 5' end, an imperfect GU repeat (40 nucleotides), and two to five upstream, out-of-frame AUG codons (Fig. 1A).

Although the banding pattern observed in cerebellar RNA was similar to that in cortical RNA in the RNase protection assays, longer transcripts appeared to predominate in the cerebellum (Fig. $2 C$ ). To address this further, an RNase protection probe, probe D (Fig. 2A), was designed that summates all transcripts -360 bases and longer into a single protected band. This probe is also partially protected by the major -340 transcript population. By hybridizing this RNA probe to total cortical and cerebellar RNA derived from each of four rats in separate reactions, we quantified the amount of long GluR2 transcripts ( -360 and longer) relative to the amount of short GluR2 transcripts $(-340)$ as shown in Figure 2D. The ratio of long to short GluR2 transcripts was greater in the cerebellum than in the cortex in each of the four rats tested (Fig. $2 D ; p<0.05$, paired $t$ test). These results indicate that GluR2 initiation site usage differs between these two brain regions and in a strict sense supports the conclusion that the GluR2 gene contains multiple promoters.

We have designated the $5^{\prime}$-most dominant transcription initiation site, approximately -429 bases from the GluR2 AUG, the +1 initiation site. All plasmid constructs used in evaluating GluR2 promoter activity by transient transfections are defined relative to this site. Our designated +1 nucleotide for the rat gene is in agreement with the $5^{\prime}$-most start site identified for the mouse GluR2 gene (Köhler et al., 1994), but there is clear evidence of minor transcriptional initiation sites further upstream and additional major sites downstream; indeed, 5' initiation sites are dispersed over $>141$ bp of the GluR2 promoter region.

The GluR2 promoter region displays several areas of high 

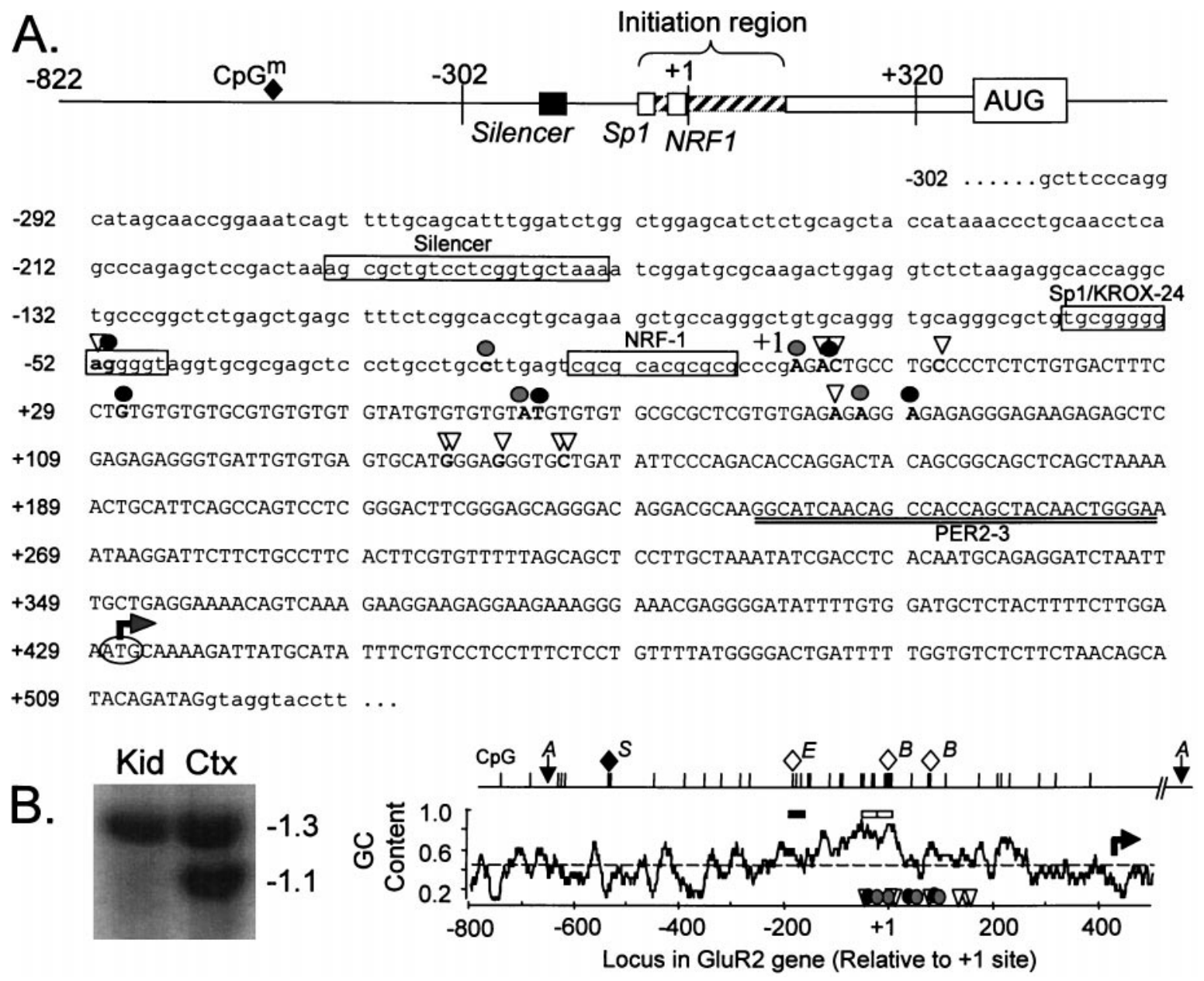

Figure 1. Features of the rat GluR2 promoter and 5'-flanking region. $A$, Schematic showing the salient features of the GluR2 promoter region and partial nucleotide sequence of the GluR2 $5^{\prime}$ proximal promoter region, exon 1 , and part of intron 1 . The exon 1 sequence is in uppercase with the $5^{\prime}$ promoter and intron 1 sequences in lowercase letters. mRNA $5^{\prime}$ ends identified by $5^{\prime}$ RACE analysis are indicated by open triangles, and those identified by RNase protection and primer extension are indicated by solid or gray circles, respectively. The $5^{\prime}$-most prominent initiation site has been designated as the +1 transcription initiation site, located 429 bases $5^{\prime}$ of the GluR2 AUG (bent arrow). Consensus cis element sequences for the GluR2 silencer element and Sp1/Krox-24 and NRF-1 transcription factors are identified by name adjacent to the corresponding boxed sequence. The boundaries of the initiation site region and the location of the methylated $\mathrm{CpG}$ dinucleotide $\left(\mathrm{CpG}^{\mathrm{m}}\right.$; filled diamond $)$ are indicated in the schematic. The location of the antisense PER2-3 oligonucleotide used for primer extension reactions is indicated by double underlining below the nucleotide sequence. $B$, GC content of the GluR2 promoter and exon 1 and a methylation-sensitive Southern blot. The average GC content \pm 10 bp surrounding a central nucleotide was calculated and plotted along the length of the GluR2 promoter. The dashed line represents the average GC content (49.7\%) for the whole $1.35 \mathrm{~kb}$ GluR2 promoter sequence shown. Open triangles, solid circles, and gray circles refer to RACE, RNase protection, and primer extension sites, respectively, as described in $A$. The solid and open horizontal bars above the GC plot represent the location of the GluR2 silencer and the Sp1/Krox-24 and NRF-1 regions, respectively. Also shown are the locations of $\mathrm{CpG}$ dinucleotides in the GluR2 promoter. The methylation state of the CpG dinucleotides was evaluated by sensitivity to $S a c$ II, Eco47III, and BssHII restriction digestion and represented by $S$, E, and $B$, respectively. The filled diamond represents a methylated $\mathrm{CpG}$ dinucleotide in kidney and lung but not in cortex and cerebellar DNA, whereas open diamonds indicate unmethylated CpGs in the DNA from all four tissues. The blot at left is an example of an AccI- and SacII-restricted Southern blot showing tissue-specific SacII digestion in cortex $(C t x)$ but not in kidney (Kid) DNA; lung and cerebellar Southern blots are not shown. The $1.3 \mathrm{~kb}$ fragment between the two AccI sites $(A)$ was radiolabeled as probe.

(86\%) and low (14\%) GC content (Fig. 1B, right). The region of highest GC content spans $\sim 150$ bp adjacent to the +1 site, includes some of the identified transcription initiation sites, and identifies a $\mathrm{CpG}$ island that could be subject to regulation by methylation in vivo. Indeed, when genomic DNA isolated from adult rat cortex, cerebellum, lung, and kidney was codigested with $A c c \mathrm{I}$ and one of the methylation-sensitive enzymes SacII, Eco47III, or BssHII, only the SacII site at -568 bp was found methylated in a tissue-specific manner. This $\mathrm{SacII}$ site was fully methylated in kidney (Fig. $1 B, 1.3 \mathrm{~kb}$ band) and lung (data not shown) genomic DNA but only partly methylated in DNA extracted from the cortex (Fig. 1B) and cerebellum (data not shown). In contrast, Eco47III and Bss $H \mathrm{II}$ restriction sites were unmethylated in all four tissues tested (data not shown). The SacII digestion pattern in cortex may be attributable to a combination of methylated glial DNA and unmethylated neuronal DNA in the sample, or it may reflect methylation of a single GluR2 allele. Although methylation of $\mathrm{CpG}$ dinucleotides increases the informational content of genes, the biological significance of the tissue-specific methylation of the GluR2 promoter remains to be addressed.

The GC-rich proximal area also contains consensus recognition sequences for Sp1/Krox-24 and the NRF-1 transcription factors (Fig. $1 A$, box areas). The regulatory role of these cis 

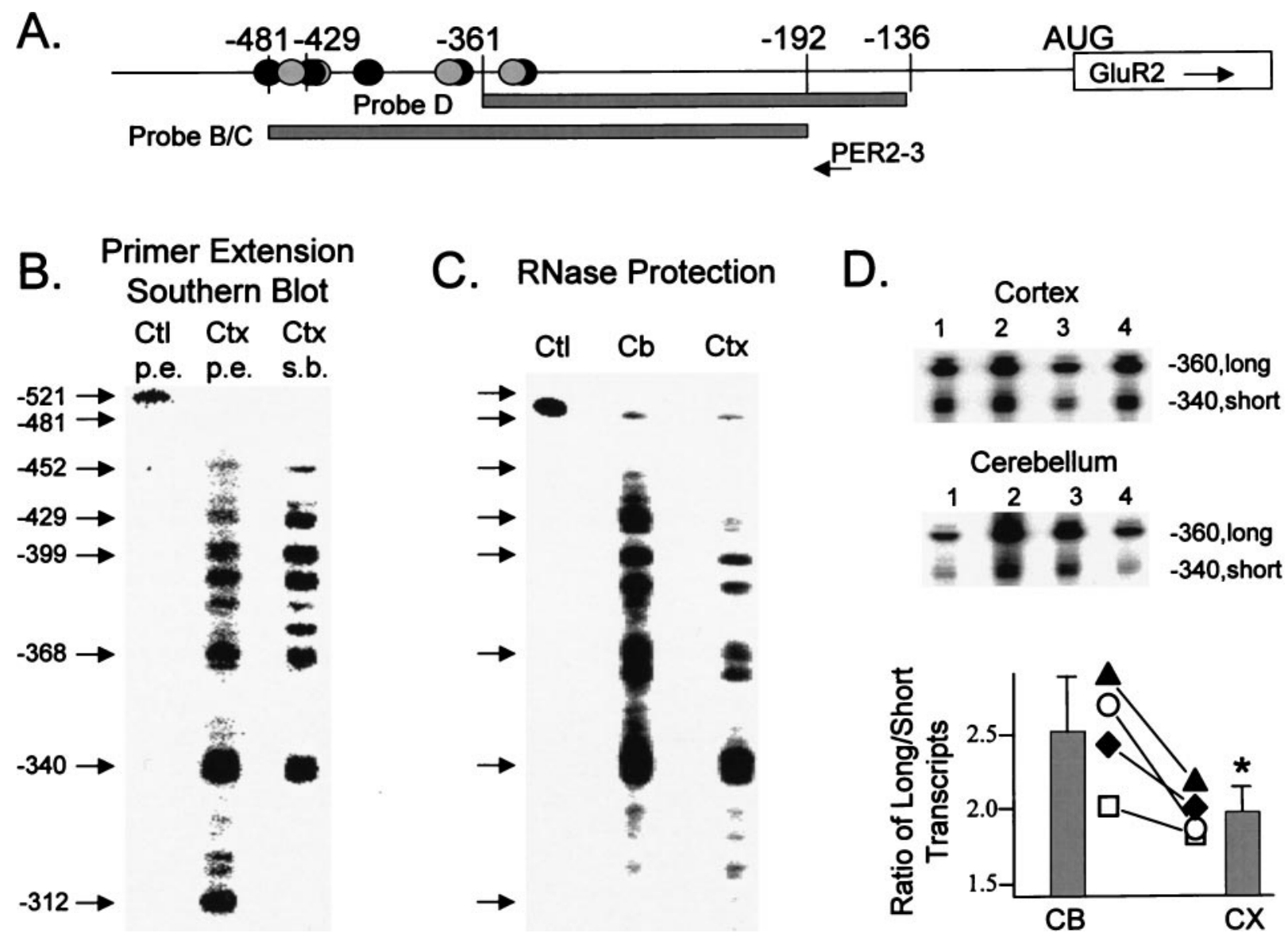

Figure 2. Differential use of transcription initiation sites in rat cortex and cerebellum. $A$, Schematic of the $5^{\prime}$ region of the GluR2 gene. Base positions indicated are relative to the GluR2 AUG codon, and identified transcription initiation sites are indicated by gray and solid circles as described in the Figure $1 A$ legend. The location of the PER2-3 oligonucleotide used for primer extension and probes B, C, and D are shown. Probes B and C encode identical sequence except probe B was a DNA probe used in the Southern blot and probe C was an antisense RNA used for RNase protection. Probe $\mathrm{D}$ was an RNA probe used for RNase protection experiments. $B$, Primer extension (p.e.) of rat cortical RNA and Southern blot (s.b.). The ${ }^{32} \mathrm{P}-\mathrm{labeled}$ PER2-3 oligonucleotide-primed extension products from $30 \mu \mathrm{g}$ of cortical RNA (middle lane) are shown. Multiple bands are observed in cortical RNA (Ctx) reactions ranging from -312 to -452 bases $5^{\prime}$ of the GluR2 AUG; none of these bands was observed in the control GluR2 in vitro synthesized RNA reactions (left lane), indicating that secondary structure does not account for the multiple bands observed in cortical RNA reactions. When the cortical primer extension products, derived from a separate reaction that used unlabeled PER2-3 oligonucleotide as primer, were Southern blotted and hybridized with probe $\mathrm{B}$, all extension products, except the shortest, were positively identified as GluR2 (right lane). C, RNase protection of $30 \mu \mathrm{g}$ of adult cortical $(C t x)$ and cerebellar $(\mathrm{Cb})$ RNA. Probe C detected multiple GluR2 5' ends both in cerebellar (middle lane) and cortical (right lane) RNA. The banding pattern shown here is nearly identical to that observed by primer extension. The $5^{\prime}$-most band at -481 relative to the AUG represents full-length probe sequence; therefore this band is a summation of all GluR2 transcripts -481 bases and longer. An RNase protection reaction to in vitro synthesized GluR2 that also extends to -481 bases is shown in the left lane (Ctl, Control). D, Regional distribution in brain of long and short GluR2 transcripts. The design of probe $\mathrm{D}$, used for RNase protection experiments against $30 \mu \mathrm{g}$ of RNA isolated from the cerebellum and cortex of each of four rats, allows for the summation of all GluR2 transcripts -360 bases and longer into a single band on the gel as well as the identification of short GluR2 transcripts that initiate at -340 bases. Results for all four rats are shown at the top and are summarized in the graph at the bottom. Lines in the graph connect the ratios of long versus short transcripts in cerebellum $(C B)$ and cortex $(C X)$ for each rat. Bar graphs are the mean \pm SEM of all data and are significantly different from each other $(p<0.05$, paired $t$ test $)$.

elements is evaluated below. Regions more distal from the +1 site exhibit a low GC content, minimal at consensus TATA box sequences at -760 and -361 bp and also at the GluR2 initiation AUG codon (Fig. 1). No TATA box element is near any of the transcriptional initiation sites identified. A weak consensus sequence for the pyrimidine-rich initiator element lies near the major 5 ' initiation site cluster at bp +3 to +11 (Javahery et al., 1994). The absence of a TATA box near transcription initiation sites is a common feature in mammalian genes, including glutamate receptor genes such as NR1, NR2B, NR2C, and KA-2 (Bai and Kusiak, 1993; Suchanek et al., 1995; Sasner and Buonanno, 1996; Huang and Gallo, 1997), and mouse GluR2 (Köhler et al., 1994). Overall, the nucleotide sequence similarity between the mouse and rat GluR2 promoter regions is high with a $90-95 \%$ sequence identity over $1.2 \mathrm{~kb}$ of the proximal promoter region,

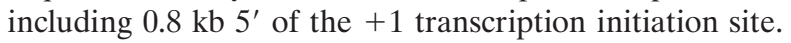

\section{Identification of a minimal promoter region}

We generated a series of $5^{\prime}$ - and $3^{\prime}$-GluR2 promoter deletion constructs and transfected these into primary cortical and primary glial cultures to define the boundaries of a minimal GluR2 promoter region and to identify cis elements that govern the cell-specific expression of GluR2. These constructs were also used to evaluate the role of consensus TATA and CAAT box sequences residing upstream in the GluR2 5' promoter. Whether deletions were made from the $5^{\prime}$ or the $3^{\prime}$ side, substantial 


\section{A. Constructs}

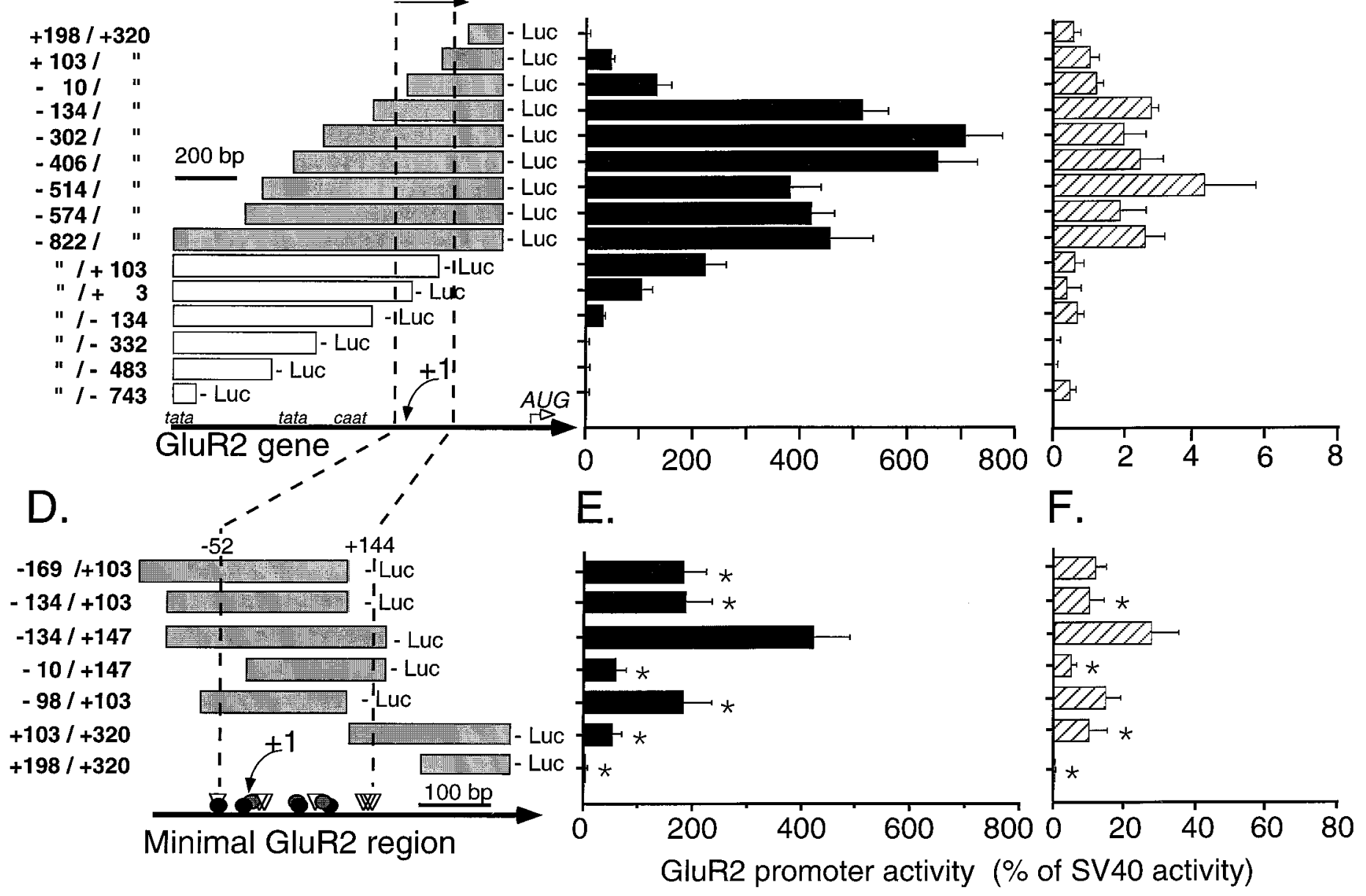

Figure 3. $5^{\prime}$ and $3^{\prime}$ deletion analysis of the GluR2 promoter. $A$, A series of 5' and $3^{\prime}$ deletion constructs was generated by PCR and cloned into the Nhe I and BglII sites of the pGL2 basic vector, upstream of firefly luciferase, for transfection into cultured cells. The boundaries of the promoter constructs are defined relative to the +1 transcriptional initiation site (Fig. 1A) and are indicated on the left, as are the relative locations of TATA and CAAT box sequences and the GluR2 AUG codon $(A ;$ bottom $)$. The area spanning all identified transcription initiation sites is indicated by the vertical dashed lines, and the +1 initiation site is marked with an arrow. $B$. Independent constructs were transfected into cortical neurons, and the promoter activity was normalized to that of SV40 to allow pooling of data from multiple experiments. When a deletion construct eliminated part of the transcription initiation site region, a significant drop in GluR2 promoter activity was observed. However, no single initiation site was essential for promoter activity. Note that these cortical neuronal cultures are a mixture of neurons $(\sim 60 \%)$ and glia $(\sim 40 \%)$. $C$, When constructs were transfected into pure cultures of primary glial cells, similar results were observed except that GluR2 promoter activity was very weak compared with that in neurons. Note the different scales of the $x$-axes in $B$ and $C$. $D$, Short promoter fragments were made by PCR to identify the minimal boundaries of the GluR2 promoter region necessary for strong neuronal expression. The locations of identified transcription initiation sites are shown with the same symbols used in Figure $1 A$. E, Transfection of minimal constructs into cultured cortical neurons shows again that elimination of part of the transcription initiation site region (dashed lines in $D$ ) significantly reduced promoter activity. $F$, Similar results were observed in parallel transfections into cultured glia, and again, promoter activity was weak compared with that in the neurons. In these glial transfections, the SV40 construct had unusually low promoter activity, resulting in an artificially high ratio of GluR2 to SV40 activity on the $x$-axis. Results shown are the mean \pm SEM for 12-33 plasmid transfections in 47 neuronal cultures in $B$, for 3-9 plasmid transfections in 17 primary glial cultures in $C$, for 7-20 plasmid transfections in 20 neuronal cultures in $E$, and for 7-20 plasmid transfections in 9 glial cultures in $F$. ${ }^{*} p<0.05$ from the R2 $(-134 /+147) l u c$ construct by ANOVA and post hoc Dunnett's tests. Statistical analysis was not conducted on data in $B$ and $C$. The average raw luciferase activity for the R2(-302/+320)luc construct in neurons and glia was $1610 \pm 160$ and $4.1 \pm 1.2$ attomoles of luciferase per $\mu \mathrm{g}$ of DNA per well, respectively.

luciferase expression was observed in primary cortical cultures when the region delimited by all transcription initiation sites $(-52$ to +144 ; Fig. $3 A$, vertical dashed lines) was included in the promoter construct (Fig. $3 B$ ). A similar pattern of promoter activity was observed when these same constructs were transfected into primary glia (Fig. 3C) or HeLa cells (data not shown), with the exception that changes in promoter activity were generally less marked and, overall, promoter strength relative to SV40 was much lower than that observed in neurons. From these experiments it seems that GluR2 promoter activity is strongly depen- dent on cis elements positioned between -134 and +320 bp (Fig. 3A,B).

To define this region of the GluR2 promoter further, we designed shorter constructs that eliminated portions of this central promoter area $(-134$ to $+320 \mathrm{bp})$, from either the $5^{\prime}$ or the $3^{\prime}$ side, and transfected these constructs into primary cortical neurons and glia. Among these, R2 $(-134 /+147) l u c$, which spanned all identified initiation sites, possessed more than twice the promoter activity of any of the shorter constructs (Fig. 3D,E). For example, when $44 \mathrm{bp}$ were removed from the $3^{\prime}$ end of 


\begin{tabular}{|c|c|c|c|c|}
\hline & Cortical neurons & Glia & C6 glioma & $\mathrm{HeLa}$ \\
\hline Promoter & \multicolumn{4}{|c|}{ [fmol of luciferase/ $\mu \mathrm{g}$ of DNA/well ( $n$ plasmid transfections)] } \\
\hline GluR2 & $1.42 \pm 0.01(80)$ & $0.014 \pm 0.001(55)$ & $0.13 \pm 0.02(44)$ & $0.27 \pm 0.02(43)$ \\
\hline SV40 & $0.28 \pm 0.03(62)$ & $4.93 \pm 1.80(32)$ & $3.27 \pm 0.77(11)$ & $0.90 \pm 0.34(29)$ \\
\hline CMV & $11.0 \pm 0.22(40)$ & $36.6 \pm 8.7(24)$ & $35.4 \pm 8.7(4)$ & $95.7 \pm 14.3(22)$ \\
\hline $\mathrm{NaII}$ & $0.11 \pm 0.03(15)$ & $5.20 \pm 0.5 \times 10^{-4}(21)$ & $0.05 \pm 0.01(21)$ & $0.07 \pm 0.03(15)$ \\
\hline Transfection efficiency $(\%)$ & $0.5-1$ & $6-8$ & $10-20$ & $10-20$ \\
\hline
\end{tabular}

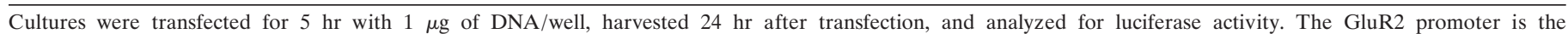

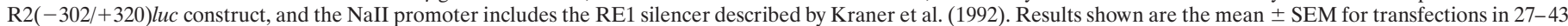

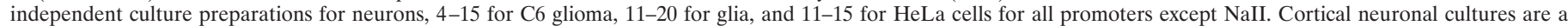

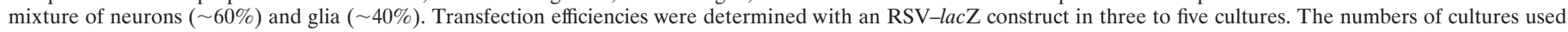
for NaII promoter transfections were five for neurons and HeLa and seven for C6 glioma and glia.

$\mathrm{R} 2(-134 /+147) l u c$ to generate $\mathrm{R} 2(-134 /+103) l u c$, promoter activity was reduced by $53 \%$ in neurons, and likewise, when $124 \mathrm{bp}$ were removed from the $5^{\prime}$ end to make R2 $(-10 /+147) l u c$, promoter activity was reduced by $86 \%$ in neurons (Fig. $3 E$ ). Similar effects of these deletions on promoter strength were observed in primary glia (Fig. 3F) and C6 glioma cells (data not shown), except promoter activity in glia was again much weaker than that in primary cortical cultures. The promoter activity of the $\mathrm{R} 2(-169 /+103)$ construct was identical to that of R2(-98/+103) (Fig. $3 D, E$ ), indicating that the sequence between -169 and -98 bp plays no role in expression under our conditions. Judging from the activities of the constructs depicted in Figure 3, it appears that dual regions within the GluR2 central promoter area may be needed for optimum expression in both cortical neuronal and glial cells. The upstream region, from -98 to $-10 \mathrm{bp}$, consists of a GC-rich sequence that includes the putative Sp1/Krox-24 and NRF-1 elements and minor initiation sites. The downstream region, from +103 to $+147 \mathrm{bp}$, includes a cluster of RACE $5^{\prime}$ ends (Fig. $1 A$ ) and a consensus sequence for metal element protein-1 (MEP-1) at bp +127 to +133 on the antisense strand. From these short constructs, we provisionally identify the entire -98 to +147 sequence as a minimal GluR2 promoter region that appears sufficient for strong expression in cortical cultures.

We also evaluated the functional role of upstream TATA box elements using constructs R2(-822/-743)luc and R2(-822/ $-332) l u c$. Both of these constructs produced little or no activity in both neurons or glia (Fig. $3 B, C$ ), eliminating a major role for the upstream TATA boxes. Similarly, inclusion of the tandem CAAT boxes at bp -317 and -324 in the R2(-822/-134)luc construct also produced very low activity in neurons and glia. These functional results support our RACE, RNase protection, and primer extension findings that failed to demonstrate an association between transcription initiation sites and upstream TATA boxes and indicate that the GluR2 gene functions as a TATA-less promoter, as suggested by Köhler et al. (1994). A construct, R2 $(-822 /+832) l u c$, which contained $0.77 \mathrm{~kb}$ of $5^{\prime}$ promoter sequence, all of exon 1, and part of the GluR2 intron 1, also showed no significant change in promoter activity in cortical cultures or primary glia (data not shown), indicating that in transiently transfected cortical cultures, the first GluR2 intron does not appear to harbor promoter regulatory elements.

\section{Neural specificity of the GluR2 promoter}

We transfected primary cortical neuronal and primary glial cultures, as well as C6 glioma and HeLa cell lines, with the R2(-302/ $+320) l u c$ construct to evaluate the neural specificity of the GluR2 promoter. Judged by the percentage of stained cells after trans- fection with a Rous sarcoma virus (RSV)- $\beta$-galactosidase expression plasmid, typical transfection efficiencies were $0.5-1 \%$ for primary neurons, $6-8 \%$ for primary glia, and $10-20 \%$ for the transformed cell lines (Table 1). Even though transfection efficiencies in the cultured neurons were low, the total raw firefly luciferase activity of the R2(-302/+320)luc construct was quite strong in these cultures, on the order of 3000 Turner light units (TLU) per $\mu \mathrm{g}$ of transfected DNA per well (background, $\sim 0.10$ TLU/well). This signal corresponds to $1.42 \pm 0.01$ fmols of luciferase per $\mu \mathrm{g}$ of DNA $(n=80)$. We estimate that in each transfection up to 9000 neurons contribute to the total signal, based on transfection into triplicate wells, $1 \times 10^{6}$ cells/well plated, an RSV-lacZ transfection efficiency of $0.5 \%$, and a $60 \%$ fractional neuronal cell population in the culture.

When luciferase activities were normalized to SV40- or the CMV-promoter activity in each experiment and the average relative activity was calculated in neurons, primary glia, C6 glioma, and HeLa cells, the R2 $(-302 /+320)$ luc construct appeared to be 37-400-fold neuronal-selective. These values, however, are overestimates caused by the preferred activities of the SV40 and CMV viral promoters in the non-neuronal cells (Table 1). Nonetheless, when viral promoter cell preferences are accounted for, the R2 $(-302 /+320) l u c$ construct still exhibited a neuronal selectivity of up to 72 -fold.

We also analyzed our data on a per-transfected-cell basis by counting the actual number of RSV-lacZ transfected cells in parallel wells stained with 5-bromo-4-chloro-3-indolyl- $\beta$ galactopyranoside. Over four experiments, the average R2(-302/ +320 ) luc promoter activity, per transfected cell, was $0.661 \pm$ 0.138 TLU in cortical cultures, $0.022 \pm 0.002 \mathrm{TLU} /$ cell in cultured glia, and $0.034 \pm 0.019 \mathrm{TLU} /$ cell in C6 glioma cells. The average ratio of cortical neuronal to glial and C6 glioma promoter activities, per cell, from all experiments, showed that GluR2 promoter activity was $32.5 \pm 3.9$ - and $26.6 \pm 8.6$-fold more active in the cortical neurons than in cortical glia and C6 glioma cells, respectively.

To provide another estimate of cell selectivity, transfected cortical neuronal cultures were costained with an anti-firefly luciferase antibody and either anti-MAP-2 or anti-GFAP antibodies $1 \mathrm{~d}$ after R2 $(-302 /+320) l u c$ transfection. Of 923 luciferasepositive cells counted, $>97 \%$ also expressed the neuron-specific marker MAP-2 (Fig. 4A,B). When immunostainings were conducted in parallel cultures to identify expression in glial cells, only 6 of 645 luciferase-positive cells costained for the glial specific marker GFAP (data not shown). In these six copositive cells, luciferase expression was marginally discernible above back- 

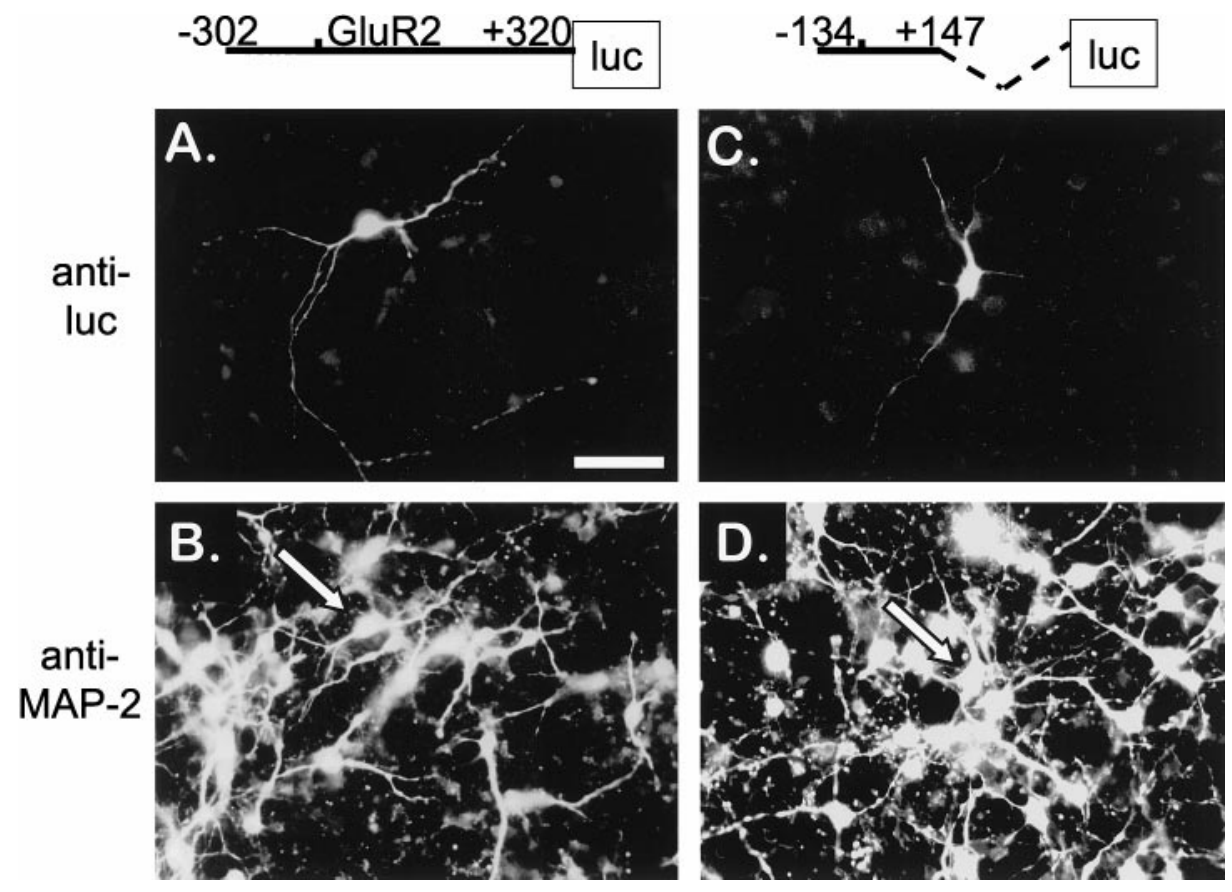

Figure 4. Minimal GluR2 promoters restrict luciferase expression to neurons. The GluR2 promoter constructs $\mathrm{R} 2(-302 /+320) l u c(A, B)$ or R2 $(-134 /+147)$ luc $(C, D)$ were transfected into day 5 primary cortical neuronal cultures and double-immunostained $24 \mathrm{hr}$ later with rabbit anti-luciferase (anti-luc) $(A, C)$ and mouse anti-MAP-2 $(B, D)$ primary antibodies as described in Materials and Methods. $A, \mathrm{~A}$ cell with neuronal morphology stained positive for luciferase expression, as detected by the goat anti-rabbit FITC-conjugated secondary antibody. $B$, The same cell in $A$ is also positive for the neuronal-specific antigen MAP-2 (arrow), detected with a donkey anti-mouse Texas Red-conjugated secondary antibody. From five $\mathrm{R} 2(-302 /+320) l u c$ transfections in which 923 luciferase-positive cells were counted, $>97 \%$ were clearly positive for the MAP-2 antigen. $C$, Another cell with neuronal morphology was positive for luciferase expression, as described in $A$, except the culture was transfected with the $\mathrm{R} 2(-134 /+147) l u c$ minimal promoter construct. $D$, The same field as in $C$ identified the transfected cell as MAP-2-positive (arrow). From two R2 $(-134 /+147)$ luc transfections in which 146 luciferase-positive cells were counted, $>93 \%$ were clearly MAP-2-positive. Scale bar: $A-D, 50 \mu \mathrm{m}$. ground fluorescence, indicating that even in these glial cells GluR2 promoter activity was weak. On the other hand, when cortical cultures were transfected with the CMV-luc construct, strong anti-luciferase staining in GFAP-positive cells was observed, indicating that transfected glial cells in the primary neuronal cultures were capable of strong luciferase expression (data not shown). These results together demonstrate that the GluR2 promoter is a strong, neuron-selective promoter that is $\sim 30$-fold stronger in primary neurons than in primary glia.

Because the GluR2 minimal promoter region also showed strong activity in neurons, we were interested in determining, by immunofluorescence, whether a minimal promoter construct could regulate cell-type-selective GluR2 expression in the mixed neuron-glia cortical cultures. When cortical cultures were transfected with R2(-134/+147)luc, $>93 \%$ of 146 luciferase-positive cells costained positive for MAP-2 (Fig. $4 C, D$ ), indicating that elements in this region of the promoter appear sufficient to guide selective luciferase expression in transfected primary cortical neurons.

\section{Deletion scan of the GluR2 promoter identifies a silencer element and a positive regulatory region}

To identify elements more precisely within the proximal promoter that regulate GluR2 expression, we made internal deletions in the R2 $(-302 /+320)$ luc construct and analyzed these by transfection into cortical neuronal and glial cultures, in parallel, as above. Most constructs substituted a unique 6 bp restriction enzyme site for 25-30 bp of GluR2 promoter sequence; the deletions in $\Delta \mathrm{K}, \Delta \mathrm{L}$, and $\Delta \mathrm{M}$ were larger (Fig. $5 B$ ). For each experiment, three or more independent plasmid DNA preparations for each construct were transfected into the cells. By this analysis, we identified a 25 bp region that, when deleted in the $\mathrm{R} 2(-302 /+320)$ luc $-\Delta \mathrm{B}$ construct, resulted in a significant increase in expression in glial cells but not in neurons (Fig. $5 B$ ). $\mathrm{R} 2(-302 /+320) l u c-\Delta \mathrm{D}$, which eliminated the -146 to $-118 \mathrm{re}-$ gion, resulted in a $47 \%$ increase in promoter activity in neurons but not in glia, suggesting that this region of the GluR2 promoter may negatively regulate expression in neurons. Three other 25-30 bp deletion constructs, $\Delta \mathrm{G}, \Delta \mathrm{H}$, and $\Delta \mathrm{I}$, caused a significant reduction in luciferase expression in both neurons and glial cells. In these cases, promoter activity in each cell type dropped by $35-65 \%$ compared with that of the parent R2 $(-302 /+320) l u c$ construct, even though the basal promoter activity differed $\sim 35$ fold in the two cultures (Fig. 5A). Results found in transfected C6 glioma cells were similar to those in glia (data not shown). The $\Delta \mathrm{G}, \Delta \mathrm{H}$, and $\Delta \mathrm{I}$ internal deletion constructs span the region from -64 to +14 bp and include the consensus sequences for Sp1/ Krox-24 and NRF-1. The $\Delta \mathrm{L}$ deletion $(+71$ to +146$)$ also resulted in a reduction in promoter activity that was marginally greater in glia than in the neuronal cultures. This deletion mutant eliminated the most $3^{\prime}$ initiation site identified by primer extension and RNase protection (Fig. $2 A, B,-340$ band), the $3^{\prime}$ cluster of RACE 5' ends, and a putative MEP-1 element. In contrast, the deletion of other major transcription initiation sites in the $\Delta \mathrm{J}, \Delta \mathrm{K}$, and $\Delta \mathrm{M}$ constructs did not reduce luciferase expression.

The pattern of expression from these internal deletion constructs complements our findings from the external $5^{\prime}$ and $3^{\prime}$ deletion series (Fig. 3) and allowed us to provisionally eliminate up to 364 bases of the GluR2 promoter from further consideration. We focused our attention on the non-neural silencer element identified in deletion $\mathrm{R} 2(-302 /+320) l u c-\Delta \mathrm{B}$ and the positive regulatory region identified in deletions $\mathrm{R} 2(-302)$ $+320) l u c-\Delta \mathrm{G},-\Delta \mathrm{H}$, and $-\Delta \mathrm{I}$.

\section{The GluR2 silencer is a cell-specific modulator of expression, not a switch}

Inspection of the GluR2 promoter sequence revealed a 21 base element with $71 \%$ identity to both the rat NaII-RE1 (Maue et al., 1990; Kraner et al., 1992) (Fig. 6A) and the rat SCG10 gene neuron-restrictive silencer element (NRSE) (Mori et al., 1992). This RE1/NRSE-like element in the GluR2 promoter is located on the antisense strand at bp -174 to -194 (Fig. 1A) and had been deleted entirely in the $\mathrm{R} 2(-302 /+320) l u c-\Delta \mathrm{B}$ construct (Fig. $5 B$ ). Given the sequence similarity, we investigated the role 

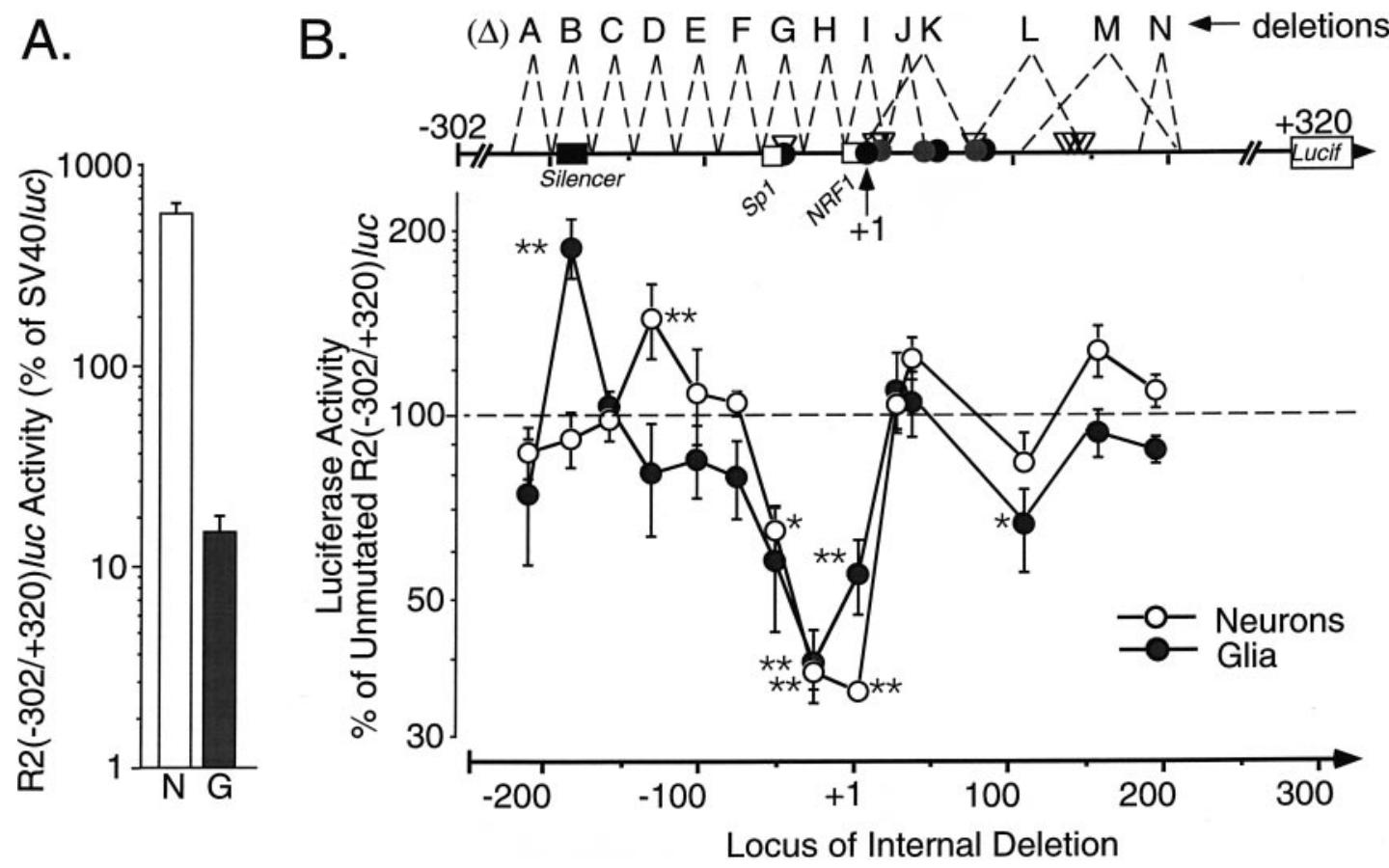

Figure 5. Mutation sensitive scan of the proximal GluR2 promoter identifies both positive and negative regulatory regions. $A$, The promoter activity of the base construct R2 $(-302 /+320) l u c$ in cultured neurons $(N ; n=63)$ and glia $(G ; n=46)$, relative to SV40luc activity, is shown. Data are the mean \pm SEM combined from all experiments. The average raw luciferase activity recovered from neurons and glia for the unmutated R2 $(-302 /+320) l u c$ control was $1320 \pm 120$ and $31 \pm 14$ attomoles per $\mu \mathrm{g}$ of DNA per well, respectively. $B$, A series of short internal deletions $(\Delta \mathrm{A}$ through $\Delta \mathrm{N}$, typically $24-31$ bp long, except $\Delta \mathrm{K}, \Delta \mathrm{L}$, and $\Delta \mathrm{M})$ were generated by replacement with unique 6 bp restriction sites in the R2 $(-302 /+320) l u c$ GluR 2 context. All deletions were generated by PCR and are shown schematically at the top. Dashed lines indicate the sequence deleted for the indicated construct (uppercase letters). Gray and solid circles and open triangles on the line indicate the locations of primer extension, RNase protection, and RACE 5' ends, respectively. Open boxes identify the locations of Sp1/Krox-24 and NRF-1 consensus sequences; the black box identifies the GluR2 silencer that is homologous with the RE1/NRSE silencers. The effect of each internal mutation on promoter activity in cultured cortical neurons and glia is shown. Luciferase activity was normalized to that of the unmutated R2 $(-302 /+320) l u c$ control construct. Note the log scale on the $y$-axis. The R2(-302/+320)luc- $\Delta \mathrm{B}$ construct resulted in a significant twofold increase in promoter activity in glia. Likewise the $\mathrm{R} 2(-302 /+320) l u c-\Delta \mathrm{D}$ deletion resulted in a significant increase in promoter activity in neurons. Three other deletions, $\mathrm{R} 2(-302 /+320) l u c-\Delta \mathrm{G},-\Delta \mathrm{H}$, and $-\Delta \mathrm{I}$, resulted in significant decreases in promoter activity in both glia and neurons. This internal deletion scan identified both a silencer region that negatively regulates GluR2 promoter activity in glia and a larger 78 bp region that positively regulates promoter activity in both neurons and glia. Data shown are the mean \pm SEM for 6-18 plasmid transfections in 21 cultured neuronal preparations and for $6-8$ transfections in 18 cultured glial preparations. lucif, Luciferase. ${ }^{*} p<0.05$ and ${ }^{* *} p<0.01$ indicate significance from the control construct by ANOVA and post hoc Dunnett's tests.

of the putative GluR2 silencer in regulating $\mathrm{R} 2(-302 /+320) l u c$ promoter activity in neuronal and non-neuronal cells. When the entire GluR2 silencer was replaced with an NsiI restriction site (Fig. $6 A, \Delta B$ ) or different 6 bp stretches were substituted by 6 bp $E c o$ RI or EcoRV restriction sites (Fig. $6 A, \delta R I, \delta R V$ ), significant two- to threefold increases in R2 $(-302 /+320) l u c$ activity were observed in glia and C6 gliomas (Fig. 6B). Similar results were observed when these same mutations were introduced into the context of a larger R2 $(-822 /+320) l u c$ promoter construct (data not shown). No changes in neuronal expression were observed by any of these mutations (Fig. 6B), demonstrating that silencing activity was specific to non-neuronal cells alone. These results are consistent with known RE1/NRSE silencers in other genes, in which short internal missense mutations are sufficient to disrupt silencing activity in non-neuronal cells (Kraner et al., 1992; Mori et al., 1992; Li et al., 1993; Mieda et al., 1997).

Because the GluR2 silencer was only $71 \%$ identical with the NaII-RE1 element and the effect of deleting this element was moderate (two- to threefold) when compared with homologous RE1/NRSE silencers (Kraner et al., 1992; Mori et al., 1992; Li et al., 1993; Wood et al., 1996; Mieda et al., 1997), we compared the ability of the GluR2 and the NaII-RE1 silencers to repress both GluR2 and NaII promoter (NaIIluc) activities in glia, C6 glioma, and neuronal cells. When the GluR2 silencer was mutated to that of the NaII-RE1 sequence in the context of the GluR2 promoter, this substitution resulted in a $30-47 \%$ decrease in promoter activity in non-neuronal cells (Fig. 6C, black bars) relative to that of the unmutated R2 $(-302 /+320)$ luc control. Conversely, when the NaII silencer in the context of the NaII promoter was mutated to that of the GluR2 silencer sequence, promoter activity was increased by $54-61 \%$ in non-neuronal cells (Fig. 6C, gray bars), indicating that repression by the NaII silencer was lessened by substitution with the GluR2 silencer. The effect of swapping these silencer elements was specific to the non-neuronal cells because no changes in neuronal expression were observed. For all of these constructs, the flanking nucleotide sequences and the orientation of the silencer element in each minimal promoter context were carefully preserved (Fig. 6C, top). Together, these results demonstrate that the NaII-RE1 silencer is more effective than the GluR2 silencer and indicate that their relative strengths are because of differences in their nucleotide sequence rather than differences in silencer orientation or in the context of the promoters in which they reside. This conclusion is further supported by the observation that both the NaII and the GluR2 minimal promoter constructs used for these experiments, in the absence of either silencer, had similar promoter activities in glia (2.0 and 

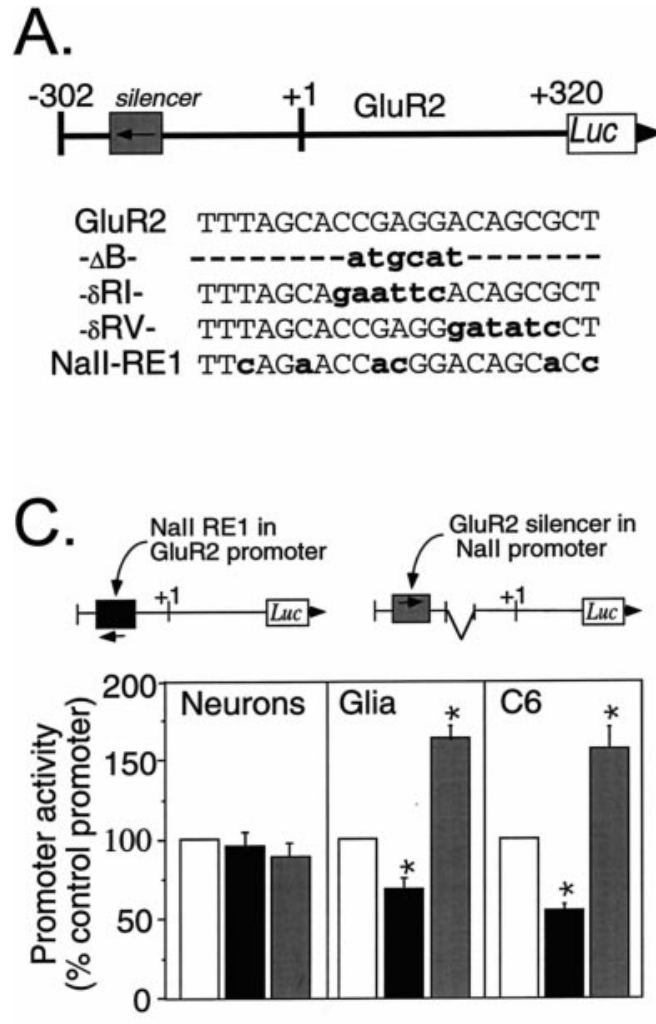

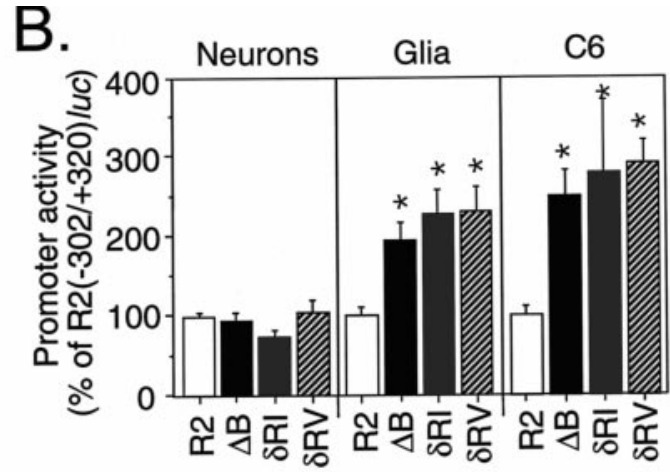

D.
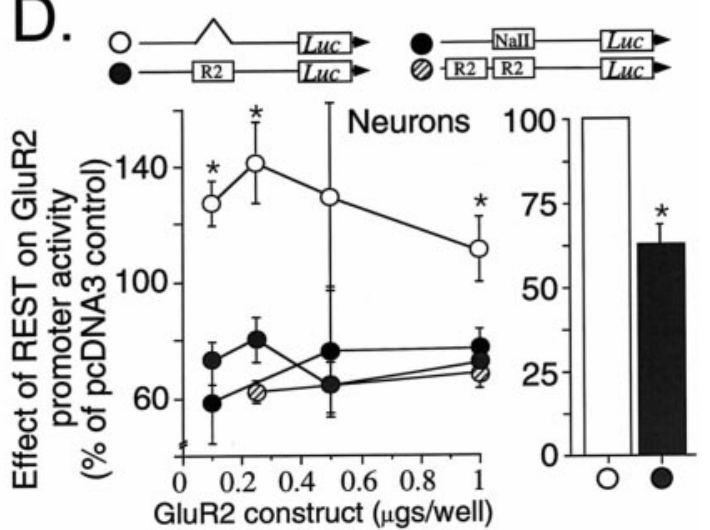

Figure 6. A weak GluR2 silencer interacts with the repressor REST. A, Schematic showing the location and antisense orientation of the silencer in the GluR2 promoter. The native (GluR2) and mutagenized silencer elements introduced into the R2 $(-302 /+320)$ luc promoter construct are listed. Dashes represent deleted sequence, and lowercase, boldface type represents substitution mutations. The $\Delta \mathrm{B}$ mutation is the same mutation as the internal deletion construct depicted in Figure 5. The NaII-RE1 sequence shown is the Type II Na channel RE1 silencer (Kraner et al., 1992), which differs from GluR2 in six positions. $B$, Functional analysis of silencer mutations on GluR2 promoter activity in primary cortical neurons, primary cortical glia, and C6 glioma cells. In neurons, no mutation resulted in a significant change in promoter activity from that of the R2 $(-302 /+320)$ luc control. In primary glia and C6 glioma cells, all three silencer mutations resulted in two- to threefold increases in GluR2 promoter activity. Data shown are the mean \pm SEM for $12-24$ plasmid transfections in neurons, 14-22 plasmid transfections in glia, and 6-34 plasmid transfections in C6 gliomas from 10, 9, and 7 culture preparations, respectively. ${ }^{*} p<0.05$ from the control by ANOVA and post hoc Dunnett's test. The error bar for the control $(R 2)$ represents the average SE among control plasmids. $C$, Promoter context independence of the GluR2 silencer. The GluR2 silencer was replaced by the NaII-RE1 silencer (see $A$ ) in the context of the R2 $(-302 /+320) l u c$ promoter. Likewise, the NaII-RE1 silencer was replaced by the GluR2 silencer in the context of the NaII promoter construct. These constructs were transfected into primary neurons, glia, and C6 glioma cells. The NaII-RE1 silencer in the context of the GluR2 promoter (black bars) reduced promoter activity by $30-47 \%$ relative to that of the unmutated R2 $(-302 /+320)$ luc control (open bars defined as $100 \%)$ in glia and C6 glioma cells. In contrast, the GluR2 silencer in the context of the NaII promoter (gray bars) increased promoter activity by 54-61\% relative to that of the unmutated NaII minimal promoter control (open bars also) in glia and C6 glioma cells. The effect of the alternative silencer in each of the promoters was significant from their respective controls; ${ }^{*} p<0.05$, Student's $t$ test. Data shown are the mean \pm SEM for $8-9$ plasmid transfections in neurons and 8-15 transfections in glia and C6 gliomas, all from three to five culture preparations for each cell type. D, Cotransfection of REST represses GluR2 promoter activity in neurons. The GluR2 silencer in the context of R2(-302/+320)luc was either unchanged (gray circles), deleted (open circles), replaced by the NaII silencer (black circles), or replaced by tandem GluR2 silencers (hatched circles) and then cotransfected into cultured cortical neurons with $0.75 \mu \mathrm{g}$ of pCMV-RESTexpress per well or the pcDNA3 empty vector. The amount of R2 $(-302 /+320) l u c$ reporter plasmid was varied from 0.1 to $1.0 \mu \mathrm{g} / \mathrm{well}$, and all tubes were balanced to $1.75 \mu \mathrm{g}$ of total DNA/well with carrier DNA (pBluescript). REST cotransfection reduced GluR2 promoter activity by $20-42 \%$ from that of the pcDNA3 control in neurons in a silencer-dependent manner. This effect by REST was observed at all concentrations of input GluR2 promoter DNA tested $(D, l e f t)$. When luciferase activities were normalized to those of the silencer-deficient construct R2(-302 +320 ) luc $-\Delta \mathrm{B}$ and pooled (right, open bar), REST coexpression reduced GluR2 promoter activity in neurons by an average of $37 \%$ (right, gray bar). Results are the mean \pm SEM for 3-14 cotransfections in 14 culture preparations. Identical results were obtained using two independent preparations of pCMV-RESTexpress, pcDNA, and pBluescript plasmid DNA. ${ }^{*} p<0.05$ or better from all other groups at the designated amount of input DNA (left, ANOVA and post hoc Dunnett's) or from the silencer-deficient construct (right, Student's $t$ test).

$3.5 \%$ of SV40, respectively) and C6 glioma cells (16.5 and 18.1\%, respectively).

We also investigated whether the GluR2 silencer could interact with a known repressor factor, REST (Chong et al., 1995), also known as neuron-restrictive silencer factor (NRSF) (Schoenherr and Anderson, 1995) or X2 box repressor (XBR) (Scholl et al., 1996), by cotransfecting the pCMV-RESTexpress plasmid with GluR2 promoter constructs into cultured neurons (Fig. 6D). There are three important considerations in the design of this cotransfection experiment. First, it is important to compare the effect of REST on normal and mutated GluR2 silencers to eliminate indirect effects of REST on other regions of the GluR2 promoter or on the expression of other endogenous genes. Second, it is necessary to cotransfect with an empty CMV vector, pcDNA3, as a control for indirect effects of the $\mathrm{CMV}$ promoter on luciferase expression. Third, it is necessary to demonstrate that simple inclusion of additional DNA in the transfection mix does not change transfection efficiency. Regarding the latter, luciferase activity was found to be linearly related to the amount of input R2luc DNA between 0.1 and $1.5 \mu \mathrm{g} /$ well, and luciferase 
activity was not influenced by adding carrier pBluescript DNA (data not shown). The cotransfection experiments are summarized in Figure $6 D$. The RESTexpress plasmid in the transfection cocktail was fixed at $0.75 \mu \mathrm{g} /$ well, the GluR2 promoter construct was varied from 0.1 to $1.0 \mu \mathrm{g} /$ well, and all tubes were balanced with carrier DNA (pBluescript) to a final mass of $1.75 \mu \mathrm{g}$ of DNA per transfected well. Compared with the pcDNA3 control, the pCMV-RESTexpress cotransfections resulted in a consistent $20-$ $42 \%$ reduction in neuronal $\mathrm{R} 2(-302 /+320)$ luc promoter activity when the reporter construct contained an intact GluR2 or NaIIRE1/NRSE silencer element (Fig. 6D, left). REST coexpression actually increased GluR2 promoter activity in neurons transfected with the GluR2 construct lacking a silencer, presumably because of an action of REST on other genes. Thus, when the data across all input DNA amounts were pooled and normalized to the activity of the construct lacking the GluR2 silencer, a significant $37 \%$ REST-mediated reduction in GluR2 promoter activity was observed (Fig. $6 D$, right). The addition of an extra GluR2 silencer element, or the substitution of the GluR2 silencer by the NaII-RE1 silencer, did not significantly enhance the effect of REST. Presumably the NaII-RE1 element was no more effective than the GluR2 silencer because $0.75 \mu \mathrm{g}$ of pCMVRESTexpress was saturating in both cotransfections. The consistent ability of REST to reduce GluR2 promoter activity in neurons in a silencer-dependent manner indicates that in intact cells the REST repressor can bind the GluR2 silencer to modify GluR2 promoter activity.

\section{Functional NRF-1 and Sp1 cis elements in the GluR2- positive regulatory region}

Based on the internal deletion scan, it was concluded that elements within the $\mathrm{R} 2(-302 /+320) l u c-\Delta \mathrm{G},-\Delta \mathrm{H}$, and $-\Delta \mathrm{I}$ deletions must contribute to GluR2 promoter activity. As shown in Figures $1 A$ and $5 B$, these regions harbor consensus sequences for $\mathrm{Sp} 1 /$ Krox-24 and NRF-1 elements and, within the $\Delta \mathrm{H}$ deletion, an element with $75 \%$ identity with the partial dyad (Patankar et al., 1997). We introduced substitution mutations into or near these elements to determine specifically whether these elements were necessary for GluR2 promoter activity in neurons. When six bases of the NRF-1 element were substituted with a BglII site (AGATCT) to generate the $\mathrm{R} 2(-302 /+320) l u c-\delta \mathrm{i} 1$ construct (Fig. $7 A$ ), a 46 and $26 \%$ drop in promoter activity was observed in cortical neurons and cortical glia, respectively (Fig. 7B, gray bars). The effect of this mutation approaches the effect observed for the larger $\mathrm{R} 2(-302 /+320) l u c-\Delta \mathrm{I}$ deletion in neurons and is consistent with the effect of mutations introduced into other NRF-1 elements (Virbasius et al., 1993; Seelan et al., 1996). The $\mathrm{R} 2(-302 /+320) l u c-\Delta \mathrm{K}$ deletion, generated by excising the sequence between two natural BssHII sites, eliminated all of the sequence in the $\Delta \mathrm{I}$ deletion except for the NRF-1 consensus element, and yet this construct did not decrease but, instead, slightly elevated promoter activity in neurons and glia (Fig. $7 B$, black bars). This result strongly suggests that the consensus NRF-1 sequence is a functional regulatory element in the GluR2 promoter. However, because the NRF-1 element is GC-rich and might therefore interact with factors other than NRF-1 (e.g., $\mathrm{Sp} 1$ ), we cotransfected the NRF-1 transcription factor (under the CMV promoter) with the unmutated R2 $(-302 /+320) l u c$ control construct and the mutated $\mathrm{R} 2(-302 /+320) l u c-\Delta \mathrm{I}$ and $-\delta \mathrm{i} 1$ constructs into neurons (Fig. $7 C$ ). NRF-1 cotransfection increased GluR2 promoter activity approximately twofold, but only when the GluR2 promoter construct contained an unmutated NRF-1 cis element (Fig. 7C). The lack of a larger effect by the cotransfected NRF-1 may be attributable to the presence of endogenous NRF-1 or some other transcription factor in the cortical neurons. These results indicate that the NRF-1 cis element sequence supports positive GluR2 promoter activity in neurons and suggests, but does not prove, that the NRF-1 transcription factor is a component of the transcriptional complex in vivo.

The NRF-1 element partially overlapped the R2(-302/ $+320) l u c-\Delta \mathrm{H}$ deletion construct (Fig. $7 \mathrm{~A}$ ), and also because the nucleotide sequence within the $\Delta \mathrm{H}$ deletion resembled the partial dyad motif described previously (Patankar et al., 1997), we introduced additional short substitution mutations into this portion of the GluR2 promoter, again in the context of $\mathrm{R} 2(-302 /+320) l u c$ (Fig. 7A). All three substitution mutations, R2(-302/+320)luc-

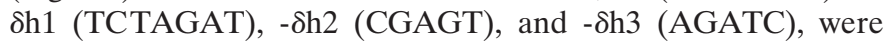
without significant effect on GluR2 promoter activity in both neurons and glia (Fig. 7B, hatched bars), indicating that neither the partial dyad nor another unidentified element in this region of the promoter contributed to GluR2 promoter activity. The strong effect of the large $\Delta \mathrm{H}$ deletion may be caused by disruption of a single base of the NRF-1 element or, more likely, caused by disruption of an appropriate architectural distance between the functional NRF-1 and Sp1/Krox-24 (see below) elements.

A third consensus element within the GluR2-positive regulatory region that we investigated by mutation analysis was the consensus Sp1 and Krox-24 sequence at -47 to -60 bp. This sequence was entirely deleted in the internal deletion R2(-302/ $+320) l u c-\Delta \mathrm{G}$ construct, resulting in a significant $35-50 \%$ reduction in promoter activity in both neurons and glia (Fig. $5 B$ ). When four bases of the consensus element were mutated (TCTA) by base substitution to generate $\mathrm{R} 2(-302 /+320) l u c-\delta \mathrm{g} 1$, a nearly identical reduction in GluR2 promoter activity was observed (Fig. $8 A, B)$, suggesting that it is the consensus $\mathrm{Sp} 1 /$ Krox-24 sequence, and not the immediate flanking sequence within the larger $\Delta \mathrm{G}$ deletion construct, that is important for GluR2 promoter activity. To differentiate between an Sp1 and Krox-24 component for GluR2 expression in neurons, we prepared nuclear extracts from cultured cortical neurons and combined these extracts with ${ }^{32} \mathrm{P}$ end-labeled double-stranded oligonucleotide probes in vitro. Oligonucleotide probes consisting of the $\Delta \mathrm{G}$ and $\Delta \mathrm{H}$ sequences shown in Figure $8 A$ produced different protein-DNA complexes in nondenaturing gel mobility shift experiments (Fig. $8 C$ ). Probe $\mathrm{G}$ formed three major complexes that were effectively competed away by 200 -fold molar excess of cold probe (compare Fig. $8 C$, lanes 2-3). Excess Sp1 oligonucleotide competed away bands 1a and $1 \mathrm{~b}$ but was less effective on band $1 \mathrm{c}$ (Fig. 8C, lane 4), and addition of an anti-Sp1 antibody to the incubations supershifted the complex, indicating that $\mathrm{Sp} 1$ is part of the protein complex recruited by probe $\mathrm{G}$ (Fig. $8 C$, arrow, lane 5). The addition of anti-Krox-24 antibody (Fig. 8C, lane 6) or competing Krox-24 oligonucleotides (Fig. 8C, lane 7) to the incubations had no effect on complex formation with probe $\mathrm{G}$. The mutant $\delta \mathrm{g} 1$ oligonucleotide was a poor competitor of $\mathrm{Sp} 1$ binding in vitro because 30-200-fold excess oligonucleotide only weakly decreased protein binding to probe $\mathrm{G}$ (Fig. 8C, lanes 18-20), compared with competition by 30-200-fold excess cold probe G DNA (Fig. 8C, lanes 15-17), consistent with the conclusion that the Sp1 element in Figure $8 A$ is the $\mathrm{Sp1}$-binding site required for functional promoter activity.

Similar in vitro binding experiments with probe $\mathrm{H}$ resulted in the formation of a single complex that was competed away with excess unlabeled probe H DNA (Fig. 8C, lane 10). This protein- 
A.
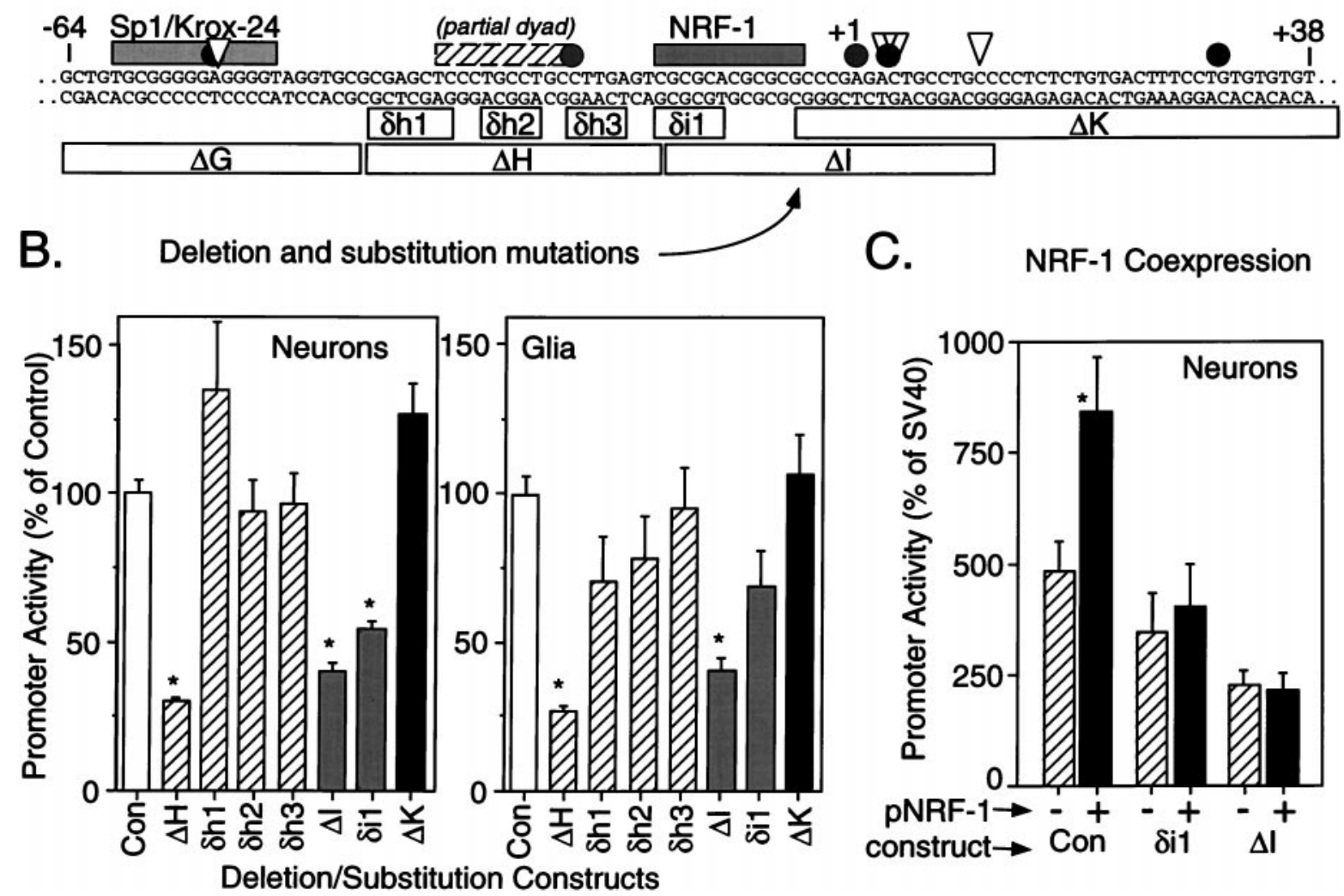

Figure 7. Contribution of the NRF-1 element to GluR2 promoter activity. $A$, Schematic of the positive regulatory domain, -64 to +38 bp, of the GluR 2 promoter region is shown with regulatory cis elements as shaded bars above the sequence; gray and solid circles and triangles signify some of the transcription initiation sites described in the Figure $1 A$ legend. Internal deletion and substitution mutations introduced into the R2(-302/+320)luc construct are shown by open bars below the sequence and identified. Deletion mutants are signified by " $\Delta$," and base-for-base substitution mutations are signified by " $\delta$." Deletion constructs are the same as those in Figure 5. B, Multiple plasmid DNA preparations for each construct were transfected into cultured cortical neurons or cortical glia, and luciferase activity was measured $1 \mathrm{~d}$ later. Promoter activity of the mutated constructs was compared with that of the R2 $(-302 /+320) l u c$ control construct $(C o n)$. The $\delta \mathrm{h} 1, \delta \mathrm{h} 2$, and $\delta \mathrm{h} 3$ substitution mutations had no effect on GluR 2 promoter activity in neurons and glia and did not replicate the effect of the larger $\Delta \mathrm{H}$ deletion construct, indicating that the effect of the $\Delta \mathrm{H}$ deletion is not dependent on nucleotide sequence (i.e., the partial dyad). In contrast, both the $\Delta \mathrm{I}$ and $\delta \mathrm{i} 1$ mutations resulted in a reduction in promoter activity in neurons and glia, whereas the $\Delta \mathrm{K}$ and $\delta \mathrm{h} 3$ constructs that modify the sequence immediately adjacent to the NRF-1 element were without effect. Together these results identify the consensus NRF-1 as a positive regulator of GluR2 promoter activity. Results shown are the mean \pm SEM for 8-12 plasmid transfections into four to five cultures. ${ }^{*} p<0.05$ from the control group by ANOVA and post hoc Dunnett's tests. $C$, One microgram of a plasmid expressing the NRF-1 transcription factor (black bars) or an empty pcDNA3 vector (cross-hatched bars) was cotransfected with $0.5 \mu \mathrm{g}$ of R2(-302/+320)luc (Con) or the $\delta \mathrm{i} 1$ or the $\Delta \mathrm{I}$ mutation constructs into neurons. Data were normalized to the activity of a parallel SV40-luc transfection in each experiment. The NRF-1 cotransfection increased promoter activity in the control construct but not in those constructs with a compromised NRF-1 cis element. Results are the mean \pm SEM in five neuronal cultures. ${ }^{*} p<0.05$ from the pcDNA3- (no NRF-1) transfected group, Student's $t$ test.

DNA complex was not disrupted by competing unlabeled probe $\mathrm{G}$ (Fig. 8C, lane 11) or Krox-24 DNA (data not shown), nor was it part of the complex supershifted by coincubation with anti-Sp1 (Fig. 8C, lane 12) or anti-Krox-24 antibodies (data not shown). The different banding patterns observed for the $\mathrm{G}$ and $\mathrm{H}$ probes as well as the nonoverlapping competition between the two probes indicate that the protein complex recruited by sequence contained within $\Delta \mathrm{G}$ of the GluR2 minimal promoter region includes Sp1.

\section{DISCUSSION}

We cloned the proximal promoter region of the rat GluR2 gene and identified three regulatory elements that govern GluR2 promoter activity in transfected cortical cultures, and we confirm the location of a major transcription initiation site $-429 /-430$ nucleotides from the AUG codon, as described previously (Köhler et al., 1994). The new conclusions of this study are (1) GluR2 transcription initiates from multiple major sites spread over $>141$ bp, (2) initiation sites are used differentially in adult cortical and cerebellar neurons, (3) GluR2 promoter activity is 30-fold stronger in cultured cortical neurons than in primary glia or C6 glioma, (4) promoter activity is regulated by both an RE1/NRSE-like silencer element and two positive regulatory elements (NRF-1 and $\mathrm{Sp} 1$ ) separated by a nucleotide bridge sequence, and (5) the GluR2 silencer is a weak silencer relative to the NaII RE1 silencer but still contributes to the neuronal specificity of the GluR2 promoter. These findings identify the first functional regulatory elements in the GluR2 promoter and point to a complex transcriptional control region for GluR2 expression in neurons.

\section{Functional implications of multiple GluR2 promoters}

Our primer extension and RNase protection results identified multiple transcription initiation sites resulting in lengthy (340481 bases) GluR2 mRNA leader sequences. These, and additional results (Fig. 2), suggest the existence of multiple GluR2 


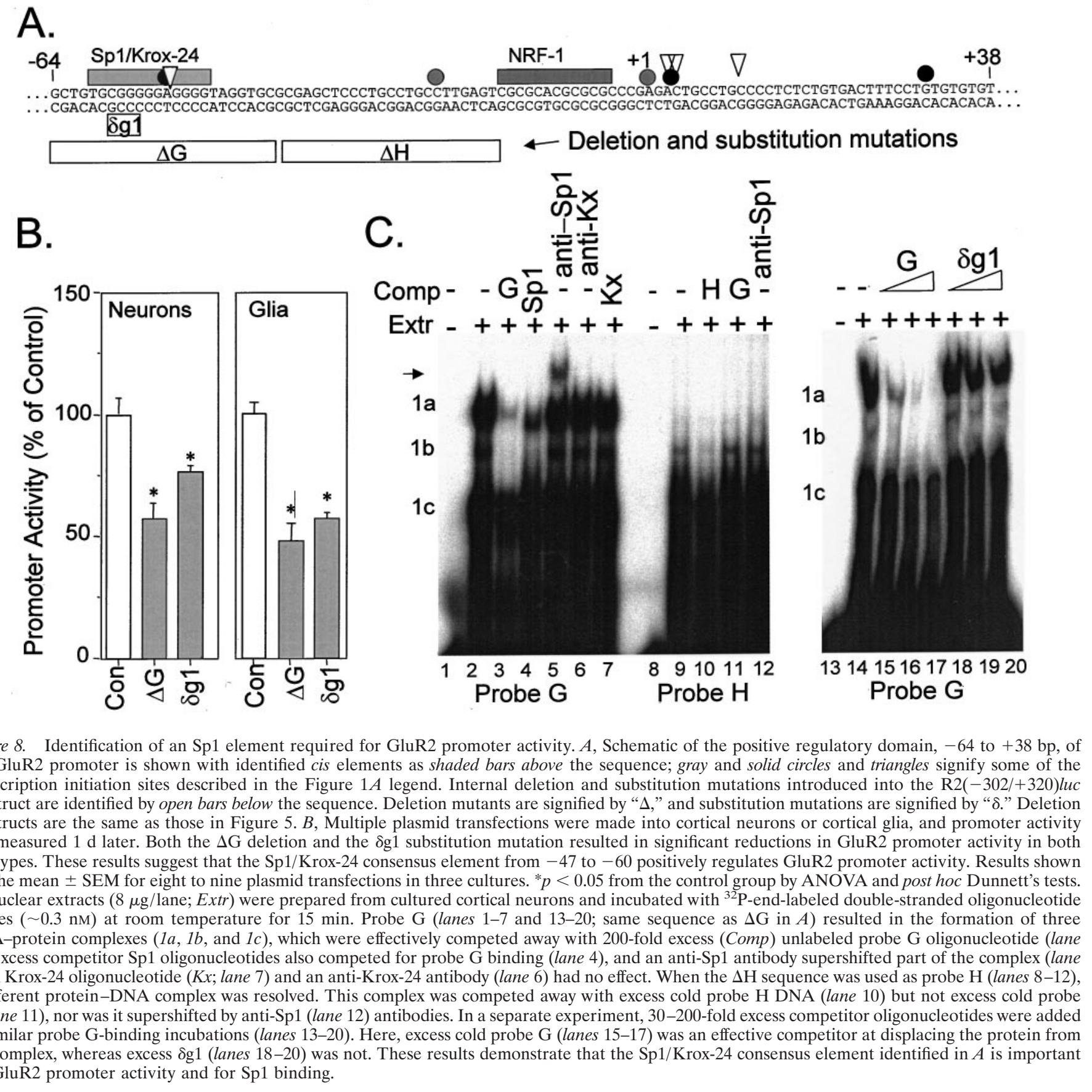

promoters. First, the observed wide distribution of transcriptional start sites $(>141 \mathrm{bp})$ is uncommon even for TATA-less promoters but is shared by other CNS genes in which multiple promoters choreograph appropriate developmental or tissue-specific gene expression (Timmusk et al., 1993; Pathak et al., 1994) or increase the complexity of mRNA transcripts within the same tissue type (Liu and Fischer, 1996). Second, we found that the number and relative use of major GluR2 transcription initiation sites in cortex were different from that in cerebellum (Fig. 2C,D). These regional differences in initiation site usage perhaps reflect the greater variety of neuronal cell types found in cerebral cortex versus cerebellum and that distinct subpopulations of cortical neurons may transcribe GluR2 from different initiation sites. Alternatively, transcription initiation could occur at multiple sites in each cortical neuron, thereby increasing the variety of GluR2 transcripts expressed per cell. In either case, the usage of the upstream transcriptional start sites is cell-selective, consistent with multiple promoters. Thirdly, no single initiation site was completely essential for GluR2 promoter activity based on our internal deletion scan (Fig. 5), indicating that RNA polymerase can initiate GluR2 transcription from multiple, independent sites. Depending on the site of transcription initiation, GluR2 mRNAs will contain variably long GC-rich 5'-UTR leaders, an imperfect GU repeat (40 nucleotides), and from two to five upstream out-of-frame AUG codons. Unusually long 5'-UTR sequences (Kozak, 1987) with high GC content and upstream AUG codons can interfere with appropriate mRNA translation (see Geballe and Morris, 1994). Indeed, we have found that sequences in the $5^{\prime}$ 
ends of the longest GluR2 transcripts profoundly impair translation in oocytes and in a cell-free translation assay (Myers et al., 1997), further emphasizing the importance of determining the cell types and conditions under which the various upstream start sites are used. Thus, whether the GluR2 mRNA is translationally suppressed is determined in part by transcriptional regulation.

\section{Modulation of GluR2 expression by an RE1/NRSE-like silencer}

The neural-specific expression of the type II sodium channel and SCG10 genes is controlled primarily by a multizinc finger repressor, termed REST or NRSF, that binds to RE1/NRSE silencer elements in their $5^{\prime}$ promoter regions. REST/NRSF expression is expressed in non-neural cells (Kraner et al., 1992, Chong et al., 1995; Schoenherr and Anderson, 1995) but also has been detected by in situ hybridization in neurons in brain including cortex (Palm et al., 1998). We have shown that the GluR2 5' promoter region contains a functional RE1/NRSE-like silencer that similarly represses GluR2 promoter activity specifically in non-neural cells (Fig. 6). However, in contrast to initial descriptions of dominant RE1/NRSE silencers that suppress expression by 10-fold or more (Maue et al., 1990; Kraner et al., 1992; Li et al., 1993), the GluR2 silencer only moderately represses promoter activity by less than threefold. Consistent with a previous report that compared silencers from multiple genes relating silencer activity with sequence homology to the consensus element (Schoenherr et al., 1996), our silencer-swapping experiments between GluR2 and sodium channel promoters (Fig. 6C) showed that enervation of the GluR2 silencer is attributable to nucleotide sequence differences rather than to contextual effects such as orientation, distance from initiation sites, or 5'- and 3 '-flanking sequences.

Thus the GluR2 silencer operates more like a modulator of expression than like a dominant switch as indicated for other genes (Kraner et al., 1992; Mori et al., 1992; Li et al., 1993). Under the conditions of our experiments, the GluR2 silencer plays little or no role in regulating GluR2 expression in cortical neurons because deletion of the silencer does not alter promoter activity in cultured cortical neurons. However, the GluR2 silencer remains a potential target for REST, REST-like transcription factors, or REST splice variants that are expressed in neurons basally or after seizure (Palm et al., 1998). Indeed, we have shown that REST can decrease GluR2 promoter activity in neurons when introduced by cotransfection (Fig. 6D). A recent report showed that an homologous sequence in the rat nicotinic $\beta 2$ subunit gene can operate either as an enhancer or as a silencer depending on its position in the promoter (Bessis et al., 1997), raising additional potential roles for RE1/NRSE-like elements in regulating transcription.

\section{Positive regulatory elements define a GluR2 minimal promoter region}

By transient transfection, the GluR2 promoter is 30-fold stronger in primary cortical neuronal cultures than in cortical glial cultures or C6 glioma cells. As judged by luciferase activity measurements in transfected cells, the GluR2 silencer contributes up to threefold neuronal selectivity, but shorter constructs lacking the silencer still show up to a 10 -fold neuronal preference after normalization to SV40 (Fig. $3 D-F$ ). When cortical cultures were transfected with the minimal R2 $(-134 /+147) l u c$ construct, $\sim 93 \%$ of the luciferase-immunopositive cells were MAP-2positive neurons, further indicating that the minimal promoter region exhibits strong neuronal selectivity (Fig. 4). We identified positive-acting Sp1 and NRF-1 elements in the GluR2 minimal promoter region by internal deletion and substitution mutation of candidate sites (Figs. 7, 8). Functional Sp1 elements are commonly found in TATA-less, GC-rich promoter regions and have been shown in other genes to interact with an NRF-1 element to coordinate promoter activity (Seelan et al., 1996). Sequence between the Sp1 and NRF-1 elements (labeled $\Delta \mathrm{H}$ in Figs. 7, 8) may serve as a spatial linker or interelement bridge to align transcription factors bound to the Sp1 and NRF-1 cis elements on the GluR2 gene, because deletion of the entire sequence reduced promoter activity by $\sim 75 \%$, but short substitution mutations designed to identify regulatory elements had no effect (Fig. $7 B$ ).

Functional NRF-1 elements are positioned near transcription initiation sites of genes for several rate-limiting enzymes (Chau et al., 1992; Virbasius et al., 1993) and mitochondrial genes (Virbasius et al., 1993; Seelan et al., 1996), suggesting that NRF-1 may serve to coordinate a variety of cellular processes by upregulating the expression of key proteins in metabolic pathways. In the GluR2 gene, Sp1 and NRF-1 elements also reside near major transcription initiation sites and contribute significantly to GluR2 promoter activity. Despite these findings, however, neither element showed cell-type selectivity in transient expression assays (Figs. 5, 7, 8). Because Sp1 and NRF-1 transcription factors are expressed in a variety of tissues including brain (Hagen et al., 1992; Gopalakrishnan and Scarpulla, 1995), the lack of neural specificity observed in these assays may not be unexpected. A functional element in the promoter of the brain-specific aldolase $\mathrm{C}$ gene has been shown to bind non-neural transcription factors in vitro yet was still able to mediate brain-specific expression in transgenic mice (Thomas et al., 1995).

Minimal promoter regions are generally defined as short sequences that are sufficient for basal promoter activity but do not regulate expression in a cell-specific manner. However, minimal promoters of GluR2, GAP-43, MAP-1B, and brain-specific aldolase $C$ genes seem to direct neural-specific expression in primary neurons, neuronal cell lines, and transgenic mice (Nedivi et al., 1992; Thomas et al., 1995; Liu and Fischer, 1996; Weber and Skene, 1997). Two major mechanisms conferring neural-specific gene expression have been proposed. First, neural specificity can be achieved by the actions of a distant and dominant silencer, such as the RE1/NRSE, on an unselective basal promoter (Kraner et al., 1992; Mori et al., 1992), or second, neural specificity can be conferred by elements near initiation sites within a minimal promoter region (Nedivi et al., 1992; Thomas et al., 1995; Liu and Fischer, 1996; Weber and Skene, 1997). The GluR2 gene seems to use both proposed mechanisms, although the minimal promoter region seems to dominate.

How can the minimal promoter sequence consisting of $\leq 245 \mathrm{bp}$ show strong neuronal preference without a clear contribution from individual elements (Fig. 5)? One explanation may be that the gain for promoter activity is higher in neurons than in glia because of the presence of a neural-specific transcription factor, or adaptor protein, that can bind either common cis regulatory elements or the general transcription factors. For example, Sp3 and $\mathrm{Sp} 4$ are additional members of the $\mathrm{Sp} 1$ transcription factor family that bind sequence elements identical to those that Sp1 binds but are expressed predominantly in brain (Hagen et al., 1992). An alternative explanation could be that transcripts are initiated from different sites in glia than in neurons and that the translation rate or stability of GluR2 mRNA in glia is significantly reduced compared with that in neurons. These latter interpretations are, however, unlikely because even the short constructs 
lacking specific 5 ' initiation sites still showed neuronal preference (Fig. 3D-F). Finally, a neural-specific element in the GluR2 promoter may exist but reside outside the region analyzed by our internal deletion scan.

Findings from this study provide a necessary foundation for understanding the transcriptional regulation of GluR2 expression in neurons. In particular, it will be of interest to determine the biological roles of the identified Sp1, NRF-1, and silencer regulatory elements in mediating changes in GluR2 levels after ischemia and status epilepticus. These results also suggest a strategy to determine why some CNS interneurons express low levels of GluR2, affecting the properties of AMPA receptor-mediated synaptic transmission. One approach would be to determine whether appropriate neuronal expression of the NRF-1, Sp1, Sp3, Sp4, and/or REST transcription factors correlates with the GluR2 expression level in different cell populations.

\section{REFERENCES}

Bai G, Kusiak JW (1993) Cloning and analysis of the 5'flanking sequence of the rat $N$-methyl-D-aspartate receptor 1 (NMDAR1) gene. Biochim Biophys Acta 1152:197-200.

Bai G, Kusiak JW (1995) Functional analysis of the proximal 5'-flanking region of the $N$-methyl-D-aspartate receptor subunit gene, NMDAR1. J Biol Chem 270:7737-7744.

Bessis A, Champtiaux N, Chatelin L, Changeux JP (1997) The neuronrestrictive silencer element: a dual enhancer/silencer crucial for patterned expression of a nicotinic receptor gene in the brain. Proc Natl Acad Sci USA 94:5906-5911.

Bochet P, Audinat E, Lambolez B, Crépel F, Rossier J, Ino M, Tsuzuki K, Ozawa S (1994) Subunit composition at the single-cell level explains functional properties of a glutamate-gated channel. Neuron 12:383-388.

Boulter J, Hollmann M, O'Shea-Greenfield A, Hartley M, Deneris E, Maron C, Heinemann S (1990) Molecular cloning and functional expression of glutamate receptor subunit genes. Science 249:1033-1037.

Bowie D, Mayer ML (1995) Inward rectification of both AMPA and kainate subtype glutamate receptors generated by polyamine-mediated ion channel block. Neuron 15:453-462.

Burnashev N, Khodorova A, Jonas P, Helm PJ, Wisden W, Monyer H, Seeburg PH, Sakmann B (1992) Calcium-permeable AMPA-kainate receptors in fusiform cerebellar glial cells. Science 256:1566-1570.

Chau CA, Evans MJ, Scarpulla RC (1992) Nuclear respiratory factor 1 activation sites in genes encoding the $\gamma$-subunit of ATP synthase, eukaryotic initiation factor $2 \alpha$, and tyrosine aminotransferase: specific interaction of purified NRF-1 with multiple target genes. J Biol Chem 267:6999-7006.

Chirgwin JM, Przybyla AE, MacDonald RJ, Rutter WJ (1979) Isolation of biologically active ribonucleic acid from sources enriched in ribonuclease. Biochemistry 18:5294-5299.

Chong JA, Tapia-Ramirez J, Kim S, Toledo-Aral JJ, Zheng Y, Boutros MC, Altshuller YM, Frohman MA, Kraner SD, Mandel G (1995) REST: a mammalian silencer protein that restricts sodium channel gene expression to neurons. Cell 80:949-957.

Cormack B (1997) Directed mutagenesis using the polymerase chain reaction. In: Current protocols in molecular biology (Ausubel FM, Brent R, Kingston RE, Moore DD, Seidman JG, Smith JA, Struhl K, eds), pp 8.5.1-8.5.10. New York: Wiley.

Dignam JD, Lebovitz RM, Roeder RG (1983) Accurate transcription by RNA polymerase II in a soluble extract from isolated mammalian nuclei. Nucleic Acids Res 11:1475-1489.

Donevan SD, Rogawski MA (1995) Intracellular polyamines mediate inward rectification of $\mathrm{Ca}^{2+}$-permeable $\alpha$-amino-3-hydroxy-5-methyl4-isoxazolepropionic acid receptors. Proc Natl Acad Sci USA 92:9298-9302.

Fitzgerald LW, Deutch AY, Gasic G, Heinemann SF, Nestler EJ (1995) Regulation of cortical and subcortical glutamate receptor subunit expression by antipsychotic drugs. J Neurosci 15:2453-2461.

Fitzgerald LW, Ortiz J, Hamedani AG, Nestler EJ (1996) Drugs of abuse and stress increase the expression of GluR1 and NMDAR1 glutamate receptor subunits in the rat ventral tegmental area: common adaptations among cross-desensitizing agents. J Neurosci 16:274-282.
Frank L, Diemer NH, Kaiser F, Sheardown M, Rasmussen JS, Kristensen P (1995) Unchanged balance between levels of mRNA encoding AMPA glutamate receptor subtypes following global cerebral ischemia in the rat. Acta Neurol Scand 92:337-343.

Friedman LK, Pellegrini-Giampietro DE, Sperber EF, Bennett MV, Moshe SL, Zukin RS (1994) Kainate-induced status epilepticus alters glutamate and $\mathrm{GABA}_{\mathrm{A}}$ receptor gene expression in adult rat hippocampus: an in situ hybridization study. J Neurosci 14:2697-2707.

Geballe AP, Morris DR (1994) Initiation codons within 5'-leaders of RNAs as regulators of translation. Trends Biochem Sci 19:159-163.

Geiger JRP, Melcher T, Koh D-S, Sakmann B, Seeburg PH, Jonas P, Monyer H (1995) Relative abundance of subunit mRNAs determines gating and $\mathrm{Ca}^{2+}$ permeability of AMPA receptors in principle neurons and interneurons in rat CNS. Neuron 15:193-204.

Gopalakrishnan L, Scarpulla RC (1995) Structure, expression, and chromosomal assignment of the human gene encoding nuclear respiratory factor 1. J Biol Chem 270:18019-18025.

Gorter JA, Petrozzino JJ, Aronica EM, Rosenbaum DM, Opitz T, Bennett MVL, Conner JA, Zukin RS (1997) Global ischemia induces downregulation of GluR2 mRNA and increases AMPA receptormediated $\mathrm{Ca}^{2+}$ influx in hippocampal CA1 neurons of gerbil. J Neurosci 17:6179-6188.

Hagen G, Muller S, Beato G, Suske G (1992) Cloning by recognition site screening of two novel GT box binding proteins: a family of Sp1 related genes. Nucleic Acids Res 20:5519-5525.

Hollmann M, Hartley M, Heinemann S (1991) $\mathrm{Ca}^{2+}$ permeability of KA-AMPA gated glutamate receptor channels depends on subunit composition. Science 252:851-853.

Huang F, Gallo V (1997) Gene structure of the rat kainate receptor subunit KA2 and characterization of an intronic negative regulatory region. J Biol Chem 272:8618-8627.

Javahery R, Khachi A, Lo K, Zenzie-Gregory B, Smale ST (1994) DNA sequence requirements for transcriptional initiator activity in mammalian cells. Mol Cell Biol 14:116-127.

Jonas P, Racca C, Sakmann B, Seeburg P, Monyer H (1994) Differences in $\mathrm{Ca}^{2+}$ permeability of AMPA-type glutamate receptor channels in neocortical neurons caused by differential GluR-B subunit expression. Neuron 12:1281-1289.

Kamboj SK, Swanson GT, Cull-Candy SG (1995) Intracellular spermine confers rectification on rat calcium-permeable AMPA and kainate receptors. J Physiol (Lond) 486:297-303.

Kamphuis W, De Rijk TC, Talamini LM, Lopes da Silva FH (1994) Rat hippocampal kindling induces changes in the glutamate receptor mRNA expression patterns in dentate granule neurons. Eur J Neurosci 6:1119-1127.

Keinänen K, Wisden W, Sommer B, Werner P, Herb A, Verdoorn TA, Sakmann B, Seeburg PH (1990) A family of AMPA-selective glutamate receptors. Science 249:556-560.

Koh D-S, Burnashev N, Jonas P (1995) Block of native $\mathrm{Ca}^{2+}$-permeable AMPA receptors in rat brain by intracellular polyamines generates double rectification. J Physiol (Lond) 486:305-312.

Köhler M, Kornau H-C, Seeburg PH (1994) The organization of the gene for the functionally dominant $\alpha$-amino-3-hydroxy-5methylisoxazole-4-propionic acid receptor subunit GluR-B. J Biol Chem 269:17367-17370.

Kozak M (1987) An analysis of 5'-noncoding sequences from 699 vertebrate messenger mRNAs. Nucleic Acids Res 15:8125-8148.

Kraner SD, Chong JA, Tsay HJ, Mandel G (1992) Silencing the Type II sodium channel gene: a model for neural-specific gene regulation. Neuron 9:37-44.

Li L, Suzuki T, Mori N, Greengard P (1993) Identification of a functional silencer element involved in neuron-specific expression of the synapsin I gene. Proc Natl Acad Sci USA 90:1460-1464.

Liu D, Fischer I (1996) Two alternative promoters direct neuron-specific expression of the rat microtubule-associated protein $1 \mathrm{~B}$ gene. J Neurosci 16:5026-5036.

Lomeli H, Mosbacher J, Melcher T, Höger T, Geiger JRP, Kuner T, Monyer H, Higuchi M, Bach A, Seeburg P (1994) Control of kinetic properties of AMPA receptor channels by nuclear editing. Science 266:1709-1713.

Maue RA, Kraner SD, Goodman RH, Mandel G (1990) Neuron-specific expression of the rat brain Type II sodium channel gene is directed by upstream regulatory elements. Neuron 4:223-231.

Mieda M, Haga T, Saffen DW (1997) Expression of the rat m4 musca- 
rinic acetylcholine receptor gene is regulated by the neuron-restrictive silencer element/repressor element 1. J Biol Chem 272:5854-5860.

Monyer H, Seeburg PH, Wisden W (1991) Glutamate-operated channels: developmentally early and mature forms arise by alternative splicing. Neuron 6:799-810.

Mori N, Schoenherr C, Vandenbergh DJ, Anderson DJ (1992) A common silencer element in the SCG10 and Type II Na ${ }^{+}$channel genes binds a factor present in nonneuronal cells but not in neuronal cells. Neuron 9:45-54.

Myers SJ, Peters JP, Dingledine R (1996) Identification of transcriptional control elements in the neuron-selective GluR2 promoter. Soc Neurosci Abstr 22:597.

Myers SJ, Revennaugh JB, Dingledine R (1997) A translational inhibitory motif resides in the GluR2 $5^{\prime}$-untranslated leader. Soc Neurosci Abstr 23:923.

Nair SM, Werkman TR, Craig J, Finnell R, Joels M, Eberwine JH (1998) Corticosteroid regulation of ion channel conductances and mRNA levels in individual hippocampal CA1 neurons. J Neurosci 18:2685-2696.

Nedivi E, Basi GS, Akey IV, Skene JHP (1992) A neural-specific GAP-43 core promoter located between unusual DNA elements that interact to regulate its activity. J Neurosci 12:691-704.

Ortiz J, Fitzgerald LW, Charlton M, Lane S, Trevisan L, Guitart X, Shoemaker W, Duman RS, Nestler EJ (1995) Biochemical actions of chronic ethanol exposure in the mesolimbic dopamine system. Synapse 21:289-298.

Palm K, Belluardo N, Metsis M, Timmusk T (1998) Neuronal expression of zinc finger transcription factor REST/NRSF/XBR gene. J Neurosci 18:1280-1296.

Patankar S, Lazaroff M, Yoon SO, Chikaraishi DM (1997) A novel basal promoter element is required for expression of the rat tyrosine hydroxylase gene. J Neurosci 17:4076-4086.

Pathak BG, Neumann JC, Croyle ML, Lingrel JB (1994) The presence of both negative and positive elements in the 5 '-flanking sequence of the rat $\mathrm{Na}, \mathrm{K}$-ATPase $\alpha 3$ subunit gene are required for brain expression in transgenic mice. Nucleic Acids Res 22:4748-4755.

Patneau DK, Wright PW, Winters C, Mayer ML, Gallo V (1994) Glial cells of the oligodendrocyte lineage express both kainate- and AMPApreferring subtypes of glutamate receptor. Neuron 12:357-371.

Pellegrini-Giampietro DE, Bennett MVL, Zukin RS (1992a) Are $\mathrm{Ca}^{2+}$-permeable kainate/AMPA receptors more abundant in immature brain? Neurosci Lett 144:65-69.

Pellegrini-Giampietro DE, Zukin RS, Bennett MVL, Cho S, Pulsinelli WA (1992b) Switch in glutamate receptor subunit gene expression in CA1 subfield of hippocampus following global ischemia in rats. Proc Natl Acad Sci USA 144:65-69.

Pellegrini-Giampietro DE, Pulsinelli WA, Zukin RS (1994) NMDA and non-NMDA receptor gene expression following global brain ischemia in rats: effect of NMDA and non-NMDA receptor antagonists. J Neurochem 67:1067-1073

Peters J, Myers SJ, Comer M, Barthel F, Dingledine R (1995) Neuronselective DNA elements in the rat GluR2 gene. Soc Neurosci Abstr 21:81.

Petralia RS, Wenthold RJ (1992) Light and electron immunocytochemical localization of AMPA-selective glutamate receptors in the rat brain. J Comp Neurol 318:329-354.

Pollard H, Hèron A, Moreau J, Ben-Ari Y, Krestchatisky M (1993)
Alterations of the GluR-B AMPA receptor subunit flip/flop expression in kainate-induced epilepsy and ischemia. Neuroscience 57:545-554.

Prince HK, Conn PJ, Blackstone CD, Huganir RL, Levey AI (1995) Down regulation of AMPA receptor subunit GluR2 in amygdaloid kindling. J Neurochem 64:462-465.

Sasner M, Buonanno A (1996) Distinct $N$-methyl-D-aspartate receptor $2 \mathrm{~B}$ subunit gene sequences confer neural and developmental specific expression. J Biol Chem 271:21316-21322.

Sato K, Kiyama H, Tohyama M (1993) The differential expression patterns of messenger RNAs encoding non- $N$-methyl-D-aspartate glutamate receptor subunits (GluR1-4) in the rat brain. Neuroscience 52:515-539.

Schoenherr CJ, Anderson DJ (1995) The neuron-restrictive silencer factor (NRSF): a coordinate repressor of multiple neuron-specific genes. Science 267:1360-1363.

Schoenherr CJ, Paquette AJ, Anderson DJ (1996) Identification of potential target genes for the neuron-restrictive silencer factor. Proc Natl Acad Sci USA 93:9881-9886.

Scholl T, Stevens MB, Mahanta S, Strominger JL (1996) A zinc finger protein that represses transcription of the human MHC class II gene, DPA. J Immunol 156:1448-1457.

Seelan RS, Gopalakrishnan L, Scarpulla RC, Grossman LI (1996) Cytochrome $c$ oxidase subunit VIIa liver isoform: characterization and identification of promoter elements in the bovine gene. J Biol Chem 271:2112-2120.

Sommer B, Keinänen K, Verdoorn T, Wisden W, Burnashev N, Herb A, Köhler M, Takagi T, Sakmann B, Seeburg PH (1990) Flip and flop: a cell specific functional switch in glutamate-operated channels of the CNS. Science 249:1580-1585.

Suchanek B, Seeburg PH, Sprengel R (1995) Gene structure of the murine $N$-methyl-D-aspartate receptor subunit NR2C. J Biol Chem 270:41-44.

Suchanek B, Seeburg PH, Sprengel R (1997) Tissue specific control regions of the $N$-methyl-D-aspartate receptor subunit NR2C promoter. Biol Chem 378:929-934.

Swanson GT, Kamboj SK, Cull-Candy SG (1997) Single-channel properties of recombinant AMPA receptors depend on RNA editing, splice variation and subunit composition. J Neurosci 17:58-69.

Thomas M, Skala H, Kahn A, Tuy FPD (1995) Functional dissection of the brain-specific rat aldolase $\mathrm{C}$ gene promoter in transgenic mice. J Biol Chem 270:20316-20321.

Timmusk T, Palm K, Metsis M, Reintam T, Paalme V, Saarma M, Persson H (1993) Multiple promoters direct tissue-specific expression of the rat BDNF gene. Neuron 10:475-489.

Virbasius CA, Virbasius JV, Scarpulla RC (1993) NRF-1, an activator involved in nuclear-mitochondrial interactions utilizes a new DNAbinding domain conserved in a family of developmental regulators. Genes Dev 7:2431-2445.

Washburn MS, Numberger M, Zhang S, Dingledine R (1997) Differential dependence on GluR2 expression of three characteristic features of AMPA receptors. J Neurosci 17:9393-9406.

Weber JRM, Skene JHP (1997) Identification of a novel repressive element that contributes to neuron-specific gene expression. J Neurosci 17:7583-7593.

Wood IC, Roopra A, Buckley NJ (1996) Neural specific expression of the $\mathrm{m} 4$ muscarinic acetylcholine receptor gene is mediated by a RE1/ NRSE-type silencing element. J Biol Chem 271:14221-14225. 